\title{
Limits of elliptic hypergeometric integrals
}

\author{
Eric M. Rains
}

September 5, 2007

\begin{abstract}
In [16, the author proved a number of multivariate elliptic hypergeometric integrals. The purpose of the present note is to explore more carefully the various limiting cases (hyperbolic, trigonometric, rational, and classical) that exist. In particular, we show (using some new estimates of generalized gamma functions) that the hyperbolic integrals (previously treated as purely formal limits) are indeed limiting cases. We also obtain a number of new trigonometric ( $q$-hypergeometric) integral identities as limits from the elliptic level.
\end{abstract}

\section{Contents}

1 Introduction

2 Asymptotics of multiple gamma functions

3 Generalized triangle inequalities

4 Hyperbolic limits

5 Trigonometric limits

6 Rational limits

7 Classical limits

\section{Introduction}

In [16], the author proved and extended a pair of multivariate elliptic hypergeometric integrals conjectured by van Diejen and Spiridonov [4], elliptic analogues of integrals due to Gustafson [9]. In a follow-up paper [5, van Diejen and Spiridonov proved "hyperbolic" degenerations of these integrals (after the hyperbolic gamma function of Ruijsensaars [18, and results of Stokman [22] at the univariate level). Unfortunately, the asymptotic estimates they had available were insufficient to derive these hyperbolic integrals as limits of the elliptic integrals; instead, they were forced to degenerate the elliptic proofs. The objective of the present paper is to put these (and other) degenerations on a sounder footing by showing that they are indeed limiting cases of the elliptic 
integral. It is to be hoped that a better understanding of the relation between the elliptic and other integrals will lead to new results at various levels, both by clarifying how arguments at low levels extend to higher levels and by providing new results at low levels as limits of the elliptic identities. The latter hope has already been fulfilled to some extent; see, for instance, Corollary 5.5 below.

A full study of degenerations of the elliptic hypergeometric integrals is beyond the scope of the present paper. Here, we focus only on the top level of each of the five types of limit (hyperbolic, trigonometric, elliptic, rational, and classical); degenerations within each type will be deferred to future work with F. van de Bult. We also do not consider in any detail the degenerations between types; rather, we obtain each case directly as a limit from the elliptic level. (Note, however, that in several cases our estimates are sufficiently uniform that one could view certain between-level limits as special cases of the limits from the elliptic level.) Similarly, we do not consider the discrete degenerations, a.k.a. hypergeometric sums; aside from finite sums (which have already been considered), the only interesting discrete degenerations appear to arise via lower-level (e.g., hyperbolic or trigonometric) integrals. Finally, we comment that at the univariate level, there are important relations between the trigonometric and hyperbolic integrals (22], also [3]); presumably, these have analogues at the multivariate level, but the existing techniques do not appear to apply. (However, see 21] for recent progress on a slightly degenerate, but multivariate case.)

The plan of the paper is as follows. In Section 2, we define the relevant gamma functions (elliptic, hyperbolic, trigonometric, and rational), and prove a number of asymptotic results relating them. In order to make the derivation of limit integrals as simple as possible, we have tried to make these estimates as uniform as possible, and the relevant error estimates similarly strong; since our techniques also apply equally well to higher-order multiple gamma functions, we prove our estimates in that level of generality. Even for the ordinary (i.e., $r=2$ ) elliptic and hyperbolic gamma functions, our results are new, as the results in the literature were nonuniform (compare, for instance, Theorem 2.8 to its special cases Corollaries 2.5 and 2.9, which were proved (for $r=2$, and not uniform) in [18]). Corollary 2.19, a uniform version of the $q \rightarrow 1$ asymptotics of $q$-symbols, may also be of independent interest.

Section 3 considers the inequalities required to pinpoint where the integrands of interest are maximized. It turns out that these all follow from a single master inequality (Lemma 3.2), which in turn follows from an asymptotic analysis of an elliptic analogue of the Cauchy determinant. The result is an inequality stating that certain combinations of elliptic gamma functions are exponentially small unless their arguments alternate around the unit circle. It may be worth investigating other elliptic determinant and pfaffian identities to see whether they give rise to interesting inequalities.

Section 4 begins the study of limit integrals with the hyperbolic case. This case turns out to be relatively straightforward, given the estimates and inequalities already established; in each case, a standard tail-exchange argument gives the desired limit. In fact, not only do we obtain the hyperbolic integrals as limits of the corresponding elliptic integrals, but moreover obtain exponentially small error estimates. We also discuss the extra arguments required to include the case in which the integrands are multiplied by appropriate abelian functions (the interpolation and biorthogonal functions of [16]).

Section 5 considers the trigonometric (i.e., basic hypergeometric) case. It turns out that in addition to the integrals of Gustafson that originally motivated van Diejen and Spiridonov, there are additional limiting cases. 
That these should exist is suggested by the fact that in the transformations of [16], only one side typically has a straightforward limit as $p \rightarrow 0$. It turns out, however, that if one breaks the symmetry of the integrand in a suitable way, one can arrange for both sides of the transformations to have reasonable limits. As a special case (violating our rule of considering only the top level of each case), we find that not only are the ordinary Macdonald polynomials limits of the biorthogonal abelian functions, but in such a way that their orthogonality follows as well; in particular, the Macdonald "conjectures" (proved in [11]) for ordinary Macdonald polynomials are limiting cases of the corresponding identities for biorthogonal abelian functions.

The remaining two cases correspond to the case $q \rightarrow 1$ with $p$ fixed. In the rational case, considered in Section 6 , the parameters all tend to 1 , and one obtains a hybrid of the hyperbolic and trigonometric cases. In particular, to obtain the full spectrum of possibilities, one must first break the symmetry of the integrand, and then do a tail-exchange asymptotic argument. The arguments are still fairly straightforward, although the resulting estimates are much weaker.

Finally, in the classical case, considered in Section 7, the parameters behave in such a way that the integrand is exponentially small unless certain inequalities are satisfied. This gives rise to multivariate analogues of the ordinary beta integral; notably those of Dixon [6] and Selberg [19. Curiously, the most natural forms of these limits remain elliptic in nature, with the integrand involving powers of theta functions, although these can be removed with a suitable change of variables.

The author would like to thank P. Forrester, J. Stokman and F. van de Bult for motivating conversations regarding the trigonometric and hyperbolic cases, and R. Askey for suggesting the use of the modular transformation to derive classical limits (as in [13]), which led the author to consider the paper [12]; the author would also like to thank an anonymous referee for pointing out that the original version of Theorem 4.7 was badly stated. The author was supported in part by NSF Grant No. DMS-0401387.

\section{Conventions}

We use standard conventions for big $O$ notation in uniform estimates; that is, we say that $f(v, z)=O(g(v, z))$ as $v \rightarrow 0^{+}$uniformly over the region $z \in D(v)$ (where $D(v)$ is a set depending on $v$ ) if there exist constants $\delta>0$ and $C>0$ such that

$$
|f(v, z)|<C|g(v, z)|
$$

whenever $0<v<\delta, z \in D(v)$; and similarly for limits $v \rightarrow \pm \infty$ (which can be viewed as limits $\pm 1 / v \rightarrow 0^{+}$).

All logarithms are taken on the principal branch, with branch cut along the negative real axis. All powers are determined correspondingly; in particular, square roots are chosen with positive real part. (The obvious exception is $\sqrt{-1}$, which is always taken to have positive imaginary part; we refrain from denoting it by $i$ so as to retain $i$ as an indexing variable.)

Finally, for a real number $x,\{x\}$ denotes the fractional part of $x$; i.e., the unique representative in $[0,1)$ of $x+\mathbb{Z}$. 


\section{Asymptotics of multiple gamma functions}

For integers $r \geq 0$, let $\phi^{(r)}\left(w ; x ; \omega_{1}, \ldots, \omega_{r}\right)$ denote the function

$$
\phi^{(r)}\left(w ; x ; \omega_{1}, \ldots, \omega_{r}\right):=\frac{e(x w)}{\prod_{1 \leq i \leq r}\left(e\left(\omega_{i} w\right)-1\right)},
$$

where $e(x):=\exp (2 \pi \sqrt{-1} x)$, and define a family of polynomials $P_{n}^{(r)}\left(x ; \omega_{1}, \ldots, \omega_{r}\right)$ in $x$ by the Laurent series expansion

$$
\phi^{(r)}\left(w ; x ; \omega_{1}, \ldots, \omega_{r}\right)=\sum_{n \geq-r} w^{n} P_{n}^{(r)}\left(x ; \omega_{1}, \ldots, \omega_{r}\right)
$$

valid in a punctured neighborhood of $w=0$; we also set

$$
Q_{n}^{(r)}\left(x ; \omega_{1}, \ldots, \omega_{r}\right):=P_{n}^{(r+1)}\left(x ; \omega_{1}, \ldots, \omega_{r},-1\right) .
$$

By convention, if $n$ is omitted, then $n=0$. Note that these are related to the multiple Bernoulli polynomials of [12] by

$$
P_{n}^{(r)}\left(x ; \omega_{1}, \ldots, \omega_{r}\right)=\frac{(2 \pi \sqrt{-1})^{n}}{(n+r) !} B_{r, n+r}\left(x \mid \omega_{1}, \ldots, \omega_{r}\right) .
$$

Note the special cases

$$
\begin{aligned}
P^{(0)}(x ;) & =1 \\
P^{(1)}(x ; \omega) & =\frac{x-\omega / 2}{\omega} \\
P^{(2)}\left(x ; \omega_{1}, \omega_{2}\right) & =\frac{12\left(x-\omega_{1} / 2-\omega_{2} / 2\right)^{2}-\omega_{1}^{2}-\omega_{2}^{2}}{24 \omega_{1} \omega_{2}}
\end{aligned}
$$

and

$$
\begin{aligned}
Q^{(0)}(x ;) & =-(x+1 / 2) \\
Q^{(1)}(x ; \omega) & =\frac{-12(x-\omega / 2+1 / 2)^{2}+\omega^{2}+1}{24 \omega} \\
Q^{(2)}\left(x ; \omega_{1}, \omega_{2}\right) & =\frac{\left(x-\omega_{1} / 2-\omega_{2} / 2+1 / 2\right)\left(-4\left(x-\omega_{1} / 2-\omega_{2} / 2+1 / 2\right)^{2}+\omega_{1}^{2}+\omega_{2}^{2}+1\right)}{24 \omega_{1} \omega_{2}} .
\end{aligned}
$$

We will also need a third polynomial

$$
\begin{aligned}
R^{(r)}\left(x ; \omega_{1}, \ldots, \omega_{r}\right) & =Q^{(r)}\left(x ; \omega_{1}, \ldots, \omega_{r}\right)+\frac{1}{2} P^{(r)}\left(x ; \omega_{1}, \ldots, \omega_{r}\right) \\
& =\frac{Q^{(r)}\left(x ; \omega_{1}, \ldots, \omega_{r}\right)+Q^{(r)}\left(x-1 ; \omega_{1}, \ldots, \omega_{r}\right)}{2}
\end{aligned}
$$

and note

$$
\begin{aligned}
R^{(0)}(x ;) & =-x \\
R^{(1)}(x ; \omega) & =\frac{-12(x-\omega / 2)^{2}+\omega^{2}-2}{24 \omega} \\
R^{(2)}\left(x ; \omega_{1}, \omega_{2}\right) & =\frac{\left(x-\omega_{1} / 2-\omega_{2} / 2\right)\left(-4\left(x-\omega_{1} / 2-\omega_{2} / 2\right)^{2}+\omega_{1}^{2}+\omega_{2}^{2}-2\right)}{24 \omega_{1} \omega_{2}} .
\end{aligned}
$$


We can then define the hyperbolic gamma function $\Gamma_{h}^{(r)}$ as follows. For all integers $r \geq 1$, and $0<\Im(x)<$ $\sum_{1 \leq i \leq r} \Im\left(\omega_{i}\right)$,

$$
\Gamma_{h}^{(r)}\left(x ; \omega_{1}, \omega_{2}, \ldots, \omega_{r}\right):=\exp \left(\mathrm{PV} \int_{\mathbb{R}} \phi^{(r)}\left(w ; x ; \omega_{1}, \ldots, \omega_{r}\right) \frac{d w}{w}\right),
$$

where by the principal value integral $\mathrm{PV} \int_{\mathbb{R}}$ we mean the average of the integral over two contours agreeing with $\mathbb{R}$ away from 0 ; one contour passes to the left of 0 , and the other passes to the right of 0 . This differs slightly from the corresponding definitions of hyperbolic gamma functions in the literature; in particular, we have

$$
\Gamma_{h}^{(r)}\left(x ; \omega_{1}, \ldots, \omega_{r}\right)=S_{r}\left(x \mid \omega_{1}, \ldots, \omega_{r}\right)^{(-1)^{r-1}},
$$

where $S_{r}$ is the multiple sine function (see, for instance, [12]), and

$$
\Gamma_{h}^{(2)}\left(x ; \omega_{1}, \omega_{2}\right)=G\left(-\omega_{1} \sqrt{-1},-\omega_{2} \sqrt{-1} ; x-\omega_{1} / 2-\omega_{2} / 2\right)
$$

in terms of Ruijsenaars' hyperbolic gamma function [18]. Since

$$
\mathrm{PV} \int_{\mathbb{R}} \phi^{(r)}\left(w ; x ; \omega_{1}, \ldots, \omega_{r}\right) \frac{d w}{w}=\frac{1}{2} \mathrm{PV} \int_{\mathbb{R}}\left(\phi^{(r)}\left(w ; x ; \omega_{1}, \ldots, \omega_{r}\right)-\phi^{(r)}\left(-w ; x ; \omega_{1}, \ldots, \omega_{r}\right)\right) \frac{d w}{w}
$$

and

$$
\mathrm{PV} \int_{\mathbb{R}} w^{k} d w=0
$$

for integers $k<-1$, we can express this as the ordinary integral over $\mathbb{R}$ of

$$
\frac{e(x w)+(-1)^{r-1} e\left(\left(\sum_{1 \leq i \leq r} \omega_{i}-x\right) w\right)}{2 w \prod_{1 \leq i \leq r}\left(e\left(\omega_{i} w\right)-1\right)}-\sum_{1 \leq k \leq(r+1) / 2} P_{1-2 k}^{(r)}\left(x ; \omega_{1}, \ldots, \omega_{r}\right) w^{-2 k},
$$

or by symmetry as twice the integral over $[0, \infty)$, thus recovering the definitions of [18] and [12].

When $r=1$, we have

$$
\mathrm{PV} \int_{\mathbb{R}} \frac{e(w x)}{(e(w \omega)-1)} \frac{d w}{w}=\log (2 \sin (\pi x / \omega))
$$

for $0<\Im(x)<\Im(\omega)$ (the branch with value $\log (2)$ at $x=\omega / 2$ ), which then gives an analytic continuation of $\Gamma_{h}^{(1)}$ to all $x, \omega$ such that $\omega \neq 0$ :

$$
\Gamma_{h}^{(1)}(x ; \omega)=2 \sin (\pi x / \omega) .
$$

(This is a fairly standard definite integral; it can be shown, for instance, by moving the contour infinitely far to the left (for $\Im(-x / \omega)>0$; if $\Im(-x / \omega)<0$, the contour should be moved to the right, and the case $-x / \omega$ real follows by analytic continuation), and observing that the resulting sum of residues (compare Theorem 2.2 below) is

$$
\pi \sqrt{-1}(x / \omega)-\pi \sqrt{-1} / 2-\sum_{k \geq 1} e(-k x / \omega) / k=\log (2 \sin (\pi x / \omega))
$$

as required.) In general, we have

$$
\Gamma_{h}^{(r)}\left(x+\omega_{r} ; \omega_{1}, \ldots, \omega_{r}\right)=\Gamma_{h}^{(r)}\left(x ; \omega_{1}, \ldots, \omega_{r}\right) \Gamma_{h}^{(r-1)}\left(x ; \omega_{1}, \ldots, \omega_{r-1}\right)
$$


in the domain of definition, and thus by induction have a meromorphic continuation to all $x$. Since $\Gamma_{h}^{(r)}$ was defined via its logarithm, we in fact have a nearly canonical choice of branch for $\log \Gamma_{h}^{(r)}$; more precisely, for each $\omega_{0}$ with $\Im\left(\omega_{0}\right)>0$, there is a unique analytic continuation of

$$
\log \Gamma_{h}^{(r)}\left(x ; \omega_{1}, \ldots, \omega_{r}\right):=\mathrm{PV} \int_{\mathbb{R}} \phi^{(r)}\left(w ; x ; \omega_{1}, \ldots, \omega_{r}\right) \frac{d w}{w}
$$

to the set $\mathcal{C}^{(r)}\left(\omega_{0} ; \omega_{1}, \ldots, \omega_{r}\right)$ obtained from $\mathbb{C}$ by removing the countable unions of rays

$$
\omega_{0} \mathbb{R}_{\geq 0}+\sum_{1 \leq i \leq r} \omega_{i} \mathbb{Z}_{\geq 1} \quad \text { and } \quad \omega_{0} \mathbb{R}_{\leq 0}+\sum_{1 \leq i \leq r} \omega_{i} \mathbb{Z}_{\leq 0} .
$$

That is, for each zero and pole of $\Gamma_{h}^{(r)}$, we cut along a ray in the direction $\pm \omega_{0}$, as appropriate. Also of importance is the analogous analytic continuation of

$$
\log \left(\left(x / \omega_{0}\right)^{(-1)^{r}} \Gamma_{h}^{(r)}\left(x ; \omega_{1}, \ldots, \omega_{r}\right)\right)
$$

to the domain $\mathcal{C}^{\prime(r)}\left(\omega_{0} ; \omega_{1}, \cdots, \omega_{r}\right)$ which differs from $\mathcal{C}^{(r)}\left(\omega_{0} ; \omega_{1}, \ldots, \omega_{r}\right)$ only in that the ray $\omega_{0} \mathbb{R}_{\leq 0}$ has not been cut; this continuation exists since the zero/pole at 0 has been cancelled. We also by convention take $\Gamma_{h}^{(0)}(x ;)=-1$, to make the functional equation valid for $r=1$ as well. For the functional equation to hold for the logarithm, we must take

$$
\log \Gamma_{h}^{(0)}(x ;):=-\pi \sqrt{-1} \operatorname{sgn}\left(\Im\left(x / \omega_{0}\right)\right),
$$

defined on the set $\mathcal{C}^{(0)}\left(\omega_{0} ;\right)=\mathbb{C} \backslash \omega_{0} \mathbb{R}$.

Remark. Using the fact that

$$
\Gamma_{h}^{(r)}\left(c x ; c \omega_{1}, \ldots, c \omega_{r}\right)=\Gamma_{h}^{(r)}\left(x ; \omega_{1}, \ldots, \omega_{r}\right)
$$

we can further extend $\Gamma_{h}^{(r)}$ to arbitrary $\omega_{i}$, so long as there exists a constant $c$ with $\Im\left(c \omega_{i}\right)>0$ for all $i$. We will not be using this extension in the sequel, although the ability to rescale within the upper half-plane will be quite useful in the proofs.

In [12, Prop. 5], Narukawa derived a product expansion for $\Gamma_{h}^{(r)}$, based on the observation that the integral over a suitably chosen sequence of contours $\Im(w)=a$ can be made to tend to 0 as $a \rightarrow \infty$, and thus the integral expands as a sum of residues. We will need a more precise form of the bound on the integral.

Lemma 2.1. Fix $\epsilon>0$, and let $a, \Im(x)$, $\omega_{i}$ range over the domain $0<\Im(x)<\sum_{i} \Im\left(\omega_{i}\right)$, and for $1 \leq i \leq r$, $\Im\left(\omega_{i}\right)>0$ and $\left|a-\Im\left(-n / \omega_{i}\right)\right|>\epsilon$, all integers $n \geq 0$. Then

$$
\left|\int_{\Im(w)=a} \phi^{(r)}\left(w ; x ; \omega_{1}, \ldots, \omega_{r}\right) \frac{d w}{w}\right| \leq a^{-1}\left[\prod_{i} C\left(\epsilon\left|\omega_{i}\right|\right)^{-1}\right]\left[\frac{\exp (-2 \pi a \Re(x))}{2 \pi \Im(x)}+\frac{\exp \left(-2 \pi a \Re\left(x-\sum_{i} \omega_{i}\right)\right)}{2 \pi \Im\left(\sum_{i} \omega_{i}-x\right)}\right],
$$

where $C(x)=\min _{d(y, \mathbb{Z}) \geq x}|e(y)-1|$. In particular, the integral is uniformly $O(\exp (-2 \pi a \Re(x)))$ over any compact subset of the domain. 
Proof. We have the estimates

$$
\begin{aligned}
|e(w x)| & =\exp (-2 \pi \Re(w) \Im(x)) \exp (-2 \pi a \Re(x)) \\
|w|^{-1} & =1 / \sqrt{\Re(w)^{2}+a^{2}} \leq 1 / a \\
\left|e\left(w \omega_{i}\right)-1\right|^{-1} & \leq C\left(\epsilon\left|\omega_{i}\right|\right)^{-1} \\
\left|e\left(w \omega_{i}\right)-1\right|^{-1} & \leq C\left(\epsilon\left|\omega_{i}\right|\right)^{-1} \exp \left(2 \pi \Re(w) \Im\left(\omega_{i}\right)\right) \exp \left(2 \pi a \Re\left(\omega_{i}\right)\right)
\end{aligned}
$$

where we note that $\left|w \omega_{i}-n\right|>\epsilon\left|\omega_{i}\right|$. If we use the third estimate for $\Re(w)>0$ and the fourth estimate for $\Re(w)<0$, we obtain the stated bound.

Narukawa then uses the fact that for $\Re(x)>\max \left(0, \Re\left(\sum_{i} \omega_{i}\right)\right)$, the above bound tends to 0 as $a \rightarrow \infty$; for our purposes, it is more convenient to fix $a$ and obtain an asymptotic series.

Theorem 2.2. Let $a, \arg (x), \omega_{i}$ range over the domain $a>0, \Im\left(e(-\arg (x) / 2 \pi) \omega_{i}\right)>0$ for $1 \leq i \leq r$. Then as $x \rightarrow \infty$,

$$
\begin{array}{r}
-\pi \sqrt{-1} P^{(r)}\left(x ; \omega_{1}, \ldots, \omega_{r}\right)-\sum_{0<\Im(e(\arg (x) / 2 \pi) y) \leq a} \operatorname{Res}_{w=y}\left(\phi^{(r)}\left(w ; x ; \omega_{1}, \ldots, \omega_{r}\right) \frac{d w}{w}\right)+\log \Gamma_{h}^{(r)}\left(x ; \omega_{1}, \ldots, \omega_{r}\right) \\
=O(\exp (-2 \pi a|x|)),
\end{array}
$$

uniformly over any compact subset of the domain.

Proof. Clearly replacing $\arg (x)$ by $\arg \left(x-b \sum_{i} \omega_{i}\right)$ for $0<b<1$ will have no effect on the validity of the bound. We may thus take

$$
x=e(\tau)|y|+b \sum_{i} \omega_{i}
$$

Since

$$
\log \Gamma_{h}^{(r)}\left(e(\phi)|y|+b \sum_{i} \omega_{i} ; \omega_{1}, \ldots, \omega_{r}\right)=\log \Gamma_{h}^{(r)}\left(|y|+b \sum_{i} e(-\phi) \omega_{i} ; e(-\phi) \omega_{1}, \ldots, e(-\phi) \omega_{r}\right),
$$

we find that uniformity in $a, \tau, \omega_{i}$ will follow from uniformity in $a, \omega_{i}$ with $\tau=0$.

Thus assume $\Im(x)=b \sum_{i} \Im\left(\omega_{i}\right)$ for $0<b<1$. For every point in the domain, there exists $a^{\prime} \geq a$ and $\epsilon>0$ such that the previous lemma applies, and such that there are no poles with imaginary part in $\left(a, a^{\prime}\right]$; by compactness, we can cover the domain by a finite number of such choices. Since $O\left(\exp \left(-2 \pi a^{\prime} \Re(x)\right)\right)=$ $O(\exp (-2 \pi a \Re(x)))$, it suffices to consider the case $a^{\prime}=a$. The result follows by residue calculus.

Remark. Note that the cut lines for $\log \Gamma_{h}^{(r)}$ can be taken along any direction $\omega_{0}$ in the convex cone generated by $\omega_{1}, \ldots, \omega_{r}$, and the argument is valid for $x$ in the complement of the cones

$$
\omega_{1}+\cdots+\omega_{r}+\mathbb{R}_{\leq 0}\left\langle\omega_{1}, \ldots, \omega_{r}\right\rangle \text { and } \quad-\mathbb{R}_{\leq 0}\left\langle\omega_{1}, \ldots, \omega_{r}\right\rangle,
$$

and not approaching $\infty$ parallel to the boundary of the cone.

In particular, we obtain the following estimate. 
Corollary 2.3. Let $a, \arg (x), \omega_{i}$ range over the domain $0<a<\min _{i} \Im\left(-e(\arg (x) / 2 \pi) / \omega_{i}\right)$. Then as $x \rightarrow \infty$, we have the estimates

$$
\begin{aligned}
-\pi \sqrt{-1} P^{(r)}\left(x ; \omega_{1}, \ldots, \omega_{r}\right)+\log \Gamma_{h}^{(r)}\left(x ; \omega_{1}, \ldots, \omega_{r}\right) & =O(\exp (-2 \pi a|x|)), \\
\pi \sqrt{-1} P^{(r)}\left(-x ; \omega_{1}, \ldots, \omega_{r}\right)+\log \Gamma_{h}^{(r)}\left(-x ; \omega_{1}, \ldots, \omega_{r}\right) & =O(\exp (-2 \pi a|x|)),
\end{aligned}
$$

uniformly on compact subsets.

Proof. The first estimate follows immediately from the theorem. The second estimate then follows using the facts

$$
P^{(r)}\left(x ; \omega_{1}, \ldots, \omega_{r}\right)=(-1)^{r} P^{(r)}\left(\sum_{i} \omega_{i}-x ; \omega_{1}, \ldots, \omega_{r}\right)
$$

and

$$
\log \Gamma_{h}^{(r)}\left(x ; \omega_{1}, \ldots, \omega_{r}\right)=(-1)^{r-1} \log \Gamma_{h}^{(r)}\left(\sum_{i} \omega_{i}-x ; \omega_{1}, \ldots, \omega_{r}\right)
$$

Remark. For $r=2$, this result is essentially due to Ruijsenaars [18, App. 2]. In general, on the domain $\Im\left(-e(\arg (x) / 2 \pi) / \omega_{i}\right)>0$, it should be possible to improve the error term to

$$
O\left(|x|^{r-1} \exp \left(-2 \pi|x| \min _{i} \Im\left(-e(\arg (x) / 2 \pi) / \omega_{i}\right)\right),\right.
$$

by taking $a=\min _{i} \Im\left(-e(\arg (x) / 2 \pi) / \omega_{i}\right)$ and bounding the leading residues. In particular, if the poles with minimum imaginary part are all simple in the given compact subset of parameter space, then one easily has the bound

$$
O\left(\exp \left(-2 \pi|x| \min _{i} \Im\left(-e(\arg (x) / 2 \pi) / \omega_{i}\right)\right)\right)
$$

on their residues, and thus on the error term.

We will also need an extension of this to the case that one of the moduli tends to 0. For convenience, write

$$
\gamma_{h}^{(r)}\left(x ; \omega_{1}, \ldots, \omega_{r}\right)=-\pi \sqrt{-1} P^{(r)}\left(x ; \omega_{1}, \ldots, \omega_{r}\right)+\log \Gamma_{h}^{(r)}\left(x ; \omega_{1}, \ldots, \omega_{r}\right) .
$$

Theorem 2.4. Let $a, \arg (x), \omega_{i}, \alpha, \beta$ range over the domain

$$
0<a<\min _{1 \leq i \leq r-1} \Im\left(-e(\arg (x) / 2 \pi) / \omega_{i}\right), \quad \Im\left(e(-\arg (x) / 2 \pi) / \omega_{r}\right)>0 .
$$

Then as $x \rightarrow \infty, v \rightarrow 0^{+}$,

$$
\begin{array}{r}
\gamma_{h}^{(r)}\left(x+v \alpha \omega_{r} ; \omega_{1}, \ldots, \omega_{r-1}, v \omega_{r}\right)-\gamma_{h}^{(r)}\left(x+v \beta \omega_{r} ; \omega_{1}, \ldots, \omega_{r-1}, v \omega_{r}\right)-(\alpha-\beta) \gamma_{h}^{(r-1)}\left(x ; \omega_{1}, \ldots, \omega_{r-1}\right) \\
=O(v \exp (-2 \pi a|x|)),
\end{array}
$$

uniformly over compact subsets of the domain. 
Proof. For $r=1$, this is immediate, so take $r \geq 2$. As before, we may restrict our attention to the case $0<\Im(x)<\Im\left(\sum_{1 \leq i \leq r-1} \omega_{i}\right)$. Then the left-hand side can be expressed as

$$
\int_{\Re(w)=a}\left(\frac{e\left(v \alpha \omega_{r} w\right)-e\left(v \beta \omega_{r} w\right)}{e\left(v \omega_{r} w\right)-1}-(\alpha-\beta)\right) \phi^{(r-1)}\left(w ; x ; \omega_{1}, \ldots, \omega_{r-1}\right) \frac{d w}{w}
$$

The desired estimate then follows as in Lemma 2.1 using the fact that as $v \rightarrow 0^{+}$,

$$
\frac{1}{v}\left(\frac{e\left(v \alpha \omega_{r} w\right)-e\left(v \beta \omega_{r} w\right)}{e\left(v \omega_{r} w\right)-1}-(\alpha-\beta)\right)
$$

is $O(\exp (\epsilon|\Re(w)|))$ for any $\epsilon>0$, uniformly in $w$ and on compact subsets of parameter space.

Corollary 2.5. Let $x, \omega_{1}, \ldots, \omega_{r}, \alpha, \beta$ range over the domain $\Im\left(\omega_{i}\right)>0, x \in \mathcal{C}^{(r-1)}\left(\omega_{r} ; \omega_{1}, \ldots, \omega_{r-1}\right)$. Then as $v \rightarrow 0^{+}$, we have the estimate

$$
\log \frac{\Gamma_{h}^{(r)}\left(x+v \alpha \omega_{r} ; \omega_{1}, \ldots, \omega_{r-1}, v \omega_{r}\right)}{\Gamma_{h}^{(r)}\left(x+v \beta \omega_{r} ; \omega_{1}, \ldots, \omega_{r-1}, v \omega_{r}\right)}-(\alpha-\beta) \log \Gamma_{h}^{(r-1)}\left(x ; \omega_{1}, \ldots, \omega_{r-1}\right)=O(v),
$$

uniformly over compact subsets of the domain.

Proof. Since

$$
\mathcal{C}^{(r-1)}\left(\omega_{r} ; \omega_{1}, \ldots, \omega_{r-1}\right)=\mathcal{C}^{(r)}\left(\omega_{r} ; \omega_{1}, \ldots, \omega_{r}\right),
$$

there is a canonical choice of branch for the left-hand side, and the proof of Theorem 2.4 gives the desired estimate on compact subsets of the complement of the cones

$$
\omega_{1}+\cdots+\omega_{r}+\mathbb{R}_{\leq 0}\left\langle\omega_{1}, \ldots, \omega_{r}\right\rangle \text { and } \quad-\mathbb{R}_{\leq 0}\left\langle\omega_{1}, \ldots, \omega_{r}\right\rangle .
$$

Since the estimate is consistent with the functional equation for $\Gamma_{h}^{(r)}$, the result follows.

Remark. In both cases, this can be extended to a uniform asymptotic series

$\log \frac{\Gamma_{h}^{(r)}\left(x+v \alpha \omega_{r} ; \omega_{1}, \ldots, \omega_{r-1}, v \omega_{r}\right)}{\Gamma_{h}^{(r)}\left(x+v \beta \omega_{r} ; \omega_{1}, \ldots, \omega_{r-1}, v \omega_{r}\right)}=\sum_{1 \leq k \leq n} \frac{B_{k}(\alpha)-B_{k}(\beta)}{k !}\left(\omega_{r} v \frac{d}{d x}\right)^{k-1} \log \Gamma_{h}^{(r-1)}\left(x ; \omega_{1}, \ldots, \omega_{r-1}\right)+O\left(v^{n}\right)$, with error $O\left(v^{n} \exp (-2 \pi a|x|)\right)$ if also $x \rightarrow \infty$; here $B_{k}(x)$ is the $k$ th (ordinary) Bernoulli polynomial.

In fact, with care, we can give an estimate valid on the larger domain $\mathcal{C}^{\prime(r-1)}\left(\omega_{r} ; \omega_{1}, \ldots, \omega_{r-1}\right)$, and thus in particular in a neighborhood of 0 . The point is that we can identify the zeros and poles of $\Gamma_{h}^{(r)}$ and $\Gamma_{h}^{(r-1)}$ that give rise to the cut line $\omega_{r} \mathbb{R}_{\leq 0}$ above, and using the ordinary gamma function, cancel them out. The resulting asymptotics can then still be computed via Stirling's formula. It will be convenient to use a slightly renormalized form of the ordinary gamma function; we define

$$
\Gamma_{r}(x ; \omega):=\frac{\Gamma(x / \omega)}{\sqrt{2 \pi}},
$$

with the convention that $\omega=1$ if omitted, and the usual convention on multiple arguments. As a justification for this convention, note that the reflection identity for the ordinary gamma function becomes

$$
\Gamma_{r}(x, \omega-x ; \omega)=\Gamma_{h}^{(1)}(x ; \omega)^{-1} .
$$


We also let $\log \Gamma_{r}(x ; \omega)$ denote the standard branch on $\mathbb{C} \backslash \omega \mathbb{R}_{\leq 0}$, and note the following version of Stirling's formula.

Theorem 2.6. Let $x \notin \mathbb{R}_{\leq 0}$. Then for all $m \geq 1$,

$$
\log \Gamma_{r}(x)=(x \log (x)-x)-\frac{1}{2} \log (x)+\sum_{1 \leq i<m} \frac{B_{2 i} x^{1-2 i}}{2 i(2 i-1)}+O\left(d\left(x, \mathbb{R}_{\leq 0}\right)^{1-2 m}\right),
$$

uniformly in $x$ as $d\left(x, \mathbb{R}_{\leq 0}\right) \rightarrow \infty$. More generally, for $\alpha$ ranging over any compact subset of $\mathbb{C}$,

$$
\log \Gamma_{r}(x+\alpha)=(x \log (x)-x)+B_{1}(\alpha) \log (x)+\sum_{1 \leq i<m} \frac{(-1)^{i+1} B_{i+1}(\alpha)}{i(i+1) x^{i}}+O\left(d\left(x, \mathbb{R}_{\leq 0}\right)^{-m}\right),
$$

uniformly as $d\left(x, \mathbb{R}_{\leq 0}\right) \rightarrow \infty$.

Proof. The claim for general $\alpha$ follows from the claim for $\alpha=0$ by straightforward algebraic manipulation. For $\alpha=0$, we observe that by [14, $\S 8.4]$ or $[2$, Thm. 1.4.2], the error term is

$$
\frac{B_{2 m} x^{1-2 m}}{2 m(2 m-1)}-\frac{1}{2 m} \int_{0}^{\infty} \frac{B_{2 m}(\{t\})}{(x+t)^{2 m}} d t .
$$

The first term certainly has the correct asymptotics; for the second term, we have

$$
\frac{1}{2 m} \int_{0}^{\infty} \frac{B_{2 m}(\{t\})}{(x+t)^{2 m}} d t=O\left(\int_{0}^{\infty}|x+t|^{-2 m} d t\right)
$$

For $\Re(x) \geq 0,|x+t|^{-2 m} \leq\left(|x|^{2}+t^{2}\right)^{-m}$, and thus the integral has order $|x|^{1-2 m}$. For $\Re(x) \leq 0$, the integral is still bounded above by

$$
\int_{-\infty}^{\infty}\left(\Im(x)^{2}+t^{2}\right)^{-m} d x=O\left(|\Im(x)|^{1-2 m}\right) .
$$

Remark. More generally, [14, Ex. 8.4.4] gives the error term

$$
\frac{(-1)^{m+1} B_{m+1}(\alpha) x^{-m}}{2 m(2 m+1)}-\frac{1}{m+1} \int_{0}^{\infty} \frac{B_{m+1}(\{t-\alpha\})}{(x+t)^{m+1}} d t
$$

for $0 \leq \alpha \leq 1$

Corollary 2.7. Let $x, \omega, \alpha, \beta$ range over the domain $\omega \neq 0, x \in \mathbb{C} \backslash \omega \mathbb{R}_{\leq 0}$. Then as $v \rightarrow 0^{+}$,

$$
\begin{aligned}
\log \frac{\Gamma_{r}(x+v \alpha \omega ; v \omega)}{\Gamma_{r}(x+v \beta \omega ; v \omega)}= & \left(B_{1}(\alpha)-B_{1}(\beta)\right) \log (x / v \omega)+\sum_{2 \leq i<m} \frac{(-1)^{i}\left(B_{i}(\alpha)-B_{i}(\beta)\right)(x / v \omega)^{1-i}}{i(i-1)} \\
& +O\left(d\left(x, \omega \mathbb{R}_{\leq 0}\right)^{-m} v^{m}\right),
\end{aligned}
$$

uniformly in $x$ and over compacta in $\omega, \alpha, \beta$. 
Theorem 2.8. Let $x, \omega_{1}, \ldots, \omega_{r}, \alpha, \beta$ range over the domain $\Im\left(\omega_{i}\right)>0, x \in \mathcal{C}^{\prime(r-1)}\left(\omega_{r} ; \omega_{1}, \ldots, \omega_{r-1}\right)$. Then as $v \rightarrow 0^{+}$, we have the estimate

$$
\begin{aligned}
\log \frac{\Gamma_{h}^{(r)}\left(x+v \alpha \omega_{r} ; \omega_{1}, \ldots, \omega_{r-1}, v \omega_{r}\right)}{\Gamma_{h}^{(r)}\left(x+v \beta \omega_{r} ; \omega_{1}, \ldots, \omega_{r-1}, v \omega_{r}\right)} & -(-1)^{r} \log \frac{\Gamma_{r}\left(x+v \alpha \omega_{r} ; v \omega_{r}\right)}{\Gamma_{r}\left(x+v \beta \omega_{r} ; v \omega_{r}\right)} \\
& -(\alpha-\beta)\left(\log \Gamma_{h}^{(r-1)}\left(x ; \omega_{1}, \ldots, \omega_{r-1}\right)-(-1)^{r} \log \left(x / v \omega_{r}\right)\right)=O(v),
\end{aligned}
$$

uniformly over compact subsets of the domain.

Proof. We first observe that

$$
\log \Gamma_{h}^{(r)}\left(x+v \alpha \omega_{r} ; \omega_{1}, \ldots, \omega_{r-1}\right)-(-1)^{r} \log \Gamma_{r}\left(x+v \alpha \omega_{r} ; v \omega_{r}\right)
$$

and

$$
\log \Gamma_{h}^{(r-1)}\left(x ; \omega_{1}, \ldots, \omega_{r-1}\right)-(-1)^{r} \log \left(x / v \omega_{r}\right)
$$

are analytic on the given domain, and thus the overall left-hand side is analytic. Moreover, the stated estimate holds on the smaller domain $\mathcal{C}^{(r-1)}\left(\omega_{r} ; \omega_{1}, \ldots, \omega_{r-1}\right)$. Using the functional equation, we may immediately extend this to the full domain $\mathcal{C}^{\prime(r-1)}\left(\omega_{r} ; \omega_{1}, \ldots, \omega_{r-1}\right)$, except when $r=2$, where the point $x=0$ must still be excluded (since the only points related to $x=0$ via the functional equation are also in the cut set). But in that case, we may simply use Cauchy's theorem to deduce a uniform estimate on a neighborhood of 0 from a uniform estimate on the boundary of the neighborhood.

Remark. More generally one has an asymptotic series in which the coefficient of the $k$ th term depends on the $(k-1)$-st derivative of

$$
\log \Gamma_{h}^{(r-1)}\left(x ; \omega_{1}, \ldots, \omega_{r-1}\right)-(-1)^{r} \log \left(x / v \omega_{r}\right)
$$

If $r=2$, we have

$$
\lim _{x \rightarrow 0} \log \Gamma_{h}^{(2)}\left(x ; \omega_{1}, \omega_{2}\right)-\log \Gamma_{r}\left(x ; \omega_{2}\right)=\frac{\log \left(\omega_{1}\right)-\log \left(\omega_{2}\right)-\log (2 \pi)}{2},
$$

which gives rise to a nice corollary by taking $x=\beta=0$ above.

Corollary 2.9. [18] As $v \rightarrow 0^{+}$,

$$
\log \Gamma_{h}^{(2)}\left(v \alpha \omega_{2} ; \omega_{1}, v \omega_{2}\right)-\log \Gamma_{r}(\alpha)-(\alpha-1 / 2) \log \left(2 \pi v \omega_{2} / \omega_{1}\right)=O(v)
$$

uniformly over compact subsets of the region $\Im\left(\omega_{1}\right), \Im\left(\omega_{2}\right)>0, \alpha \in \mathbb{C}$.

Now, consider the elliptic gamma function, defined as

$$
\Gamma_{e}^{(r)}\left(z ; p_{1}, p_{2}, \ldots, p_{r}\right)=\prod_{0 \leq k_{1}, k_{2}, \ldots, k_{r}}\left(1-p_{1}^{k_{1}+1} p_{2}^{k_{2}+1} \cdots p_{r}^{k_{r}+1} / z\right)\left(1-p_{1}^{k_{1}} p_{2}^{k_{2}} \cdots p_{r}^{k_{r}} z\right)^{(-1)^{r-1}} .
$$

For $\left|p_{1} p_{2} \cdots p_{r}\right|<|z|<1$, we have

$$
\log \Gamma_{e}^{(r)}\left(z ; p_{1}, p_{2}, \ldots, p_{r}\right)=\sum_{1 \leq k} \frac{(-1)^{r} z^{k}-\left(p_{1} p_{2} \cdots p_{r} / z\right)^{k}}{k \prod_{1 \leq i \leq r}\left(1-p_{i}^{k}\right)}
$$


this, then, for any $\omega_{0}$ with $\Im\left(\omega_{0}\right)>0$, defines a branch of $\log \Gamma_{e}^{(r)}$ on the region $\mathcal{C}_{e}^{(r)}\left(\omega_{0} ; p_{1}, \ldots, p_{r}\right)$ obtained from $\mathbb{C}^{*}=\mathbb{C} \backslash\{0\}$ by removing the countable union of logarithmic spirals

$$
p_{1}^{\mathbb{Z}_{\geq 1}} p_{2}^{\mathbb{Z}_{\geq 1}} \cdots p_{r}^{\mathbb{Z}_{\geq 1}} e\left(\omega_{0} \mathbb{R}_{\geq 0}\right) \quad \text { and } \quad p_{1}^{\mathbb{Z}_{\leq 0}} p_{2}^{\mathbb{Z}_{\leq 0}} \cdots p_{r}^{\mathbb{Z}_{\leq 0}} e\left(\omega_{0} \mathbb{R}_{\leq 0}\right) .
$$

(We define a region $\mathcal{C}_{e}^{\prime(r)}$ analogously.) And, of course, we have the functional equation

$$
\log \Gamma_{e}^{(r)}\left(p_{r} z ; p_{1}, p_{2}, \ldots, p_{r}\right)-\log \Gamma_{e}^{(r)}\left(z ; p_{1}, p_{2}, \ldots, p_{r}\right)=\log \Gamma_{e}^{(r-1)}\left(z ; p_{1}, p_{2}, \ldots, p_{r-1}\right),
$$

with

$$
\log \Gamma_{e}^{(0)}(z ;)=\log (-1 / z), \quad \log \Gamma_{e}^{(0)}(-1 ;)=0 .
$$

Narukawa [12, Theorem 14] gives the following "product" expansion of the elliptic gamma function in terms of the hyperbolic gamma function:

$$
\begin{aligned}
-2 \pi \sqrt{-1} Q^{(r)}\left(x ; \omega_{1}, \ldots, \omega_{r}\right)+ & \log \Gamma_{e}^{(r)}\left(e(x) ; e\left(\omega_{1}\right), \ldots, e\left(\omega_{r}\right)\right) \\
& =\sum_{0 \leq k}-\pi \sqrt{-1} P^{(r)}\left(x+k+1 ; \omega_{1}, \ldots, \omega_{r}\right)+\log \Gamma_{h}^{(r)}\left(x+k+1 ; \omega_{1}, \ldots, \omega_{r}\right) \\
& +\sum_{0 \leq k} \pi \sqrt{-1} P^{(r)}\left(x-k ; \omega_{1}, \ldots, \omega_{r}\right)+\log \Gamma_{h}^{(r)}\left(x-k ; \omega_{1}, \ldots, \omega_{r}\right)
\end{aligned}
$$

Note that each term in the infinite sums converges uniformly exponentially to 0 as $k \rightarrow \infty$, so the sums converge uniformly and absolutely.

Using this expansion and the asymptotics of the hyperbolic gamma function, we obtain the following estimates for the elliptic gamma function. First, the hyperbolic limit $p_{1}, \ldots, p_{r} \rightarrow 1$.

Proposition 2.10. Let $A, \omega_{1}, \ldots, \omega_{r}, x$ range over the domain

$$
0<A<\min _{1 \leq i \leq r} \Im\left(-1 / \omega_{i}\right)-\left|\Im\left(x / \omega_{i}\right)\right| .
$$

Then as $v \rightarrow 0^{+}$, we have the estimate

$-2 \pi \sqrt{-1} R^{(r)}\left(x ; v \omega_{1}, \ldots, v \omega_{r}\right)+\log \Gamma_{e}^{(r)}\left(e(x) ; e\left(v \omega_{1}\right), \ldots, e\left(v \omega_{r}\right)\right)-\log \Gamma_{h}^{(r)}\left(x ; v \omega_{1}, \ldots, v \omega_{r}\right)=O(\exp (-2 \pi A / v))$, uniform over compact subsets of the domain.

Remark. If we do not care about the choice of $A$, the constraint on the domain is simply

$$
\left|\Im\left(x / \omega_{i}\right)\right|<\Im\left(-1 / \omega_{i}\right), 1 \leq i \leq r
$$

this is a parallelogram, two of the vertices of which are \pm 1 .

Proposition 2.11. Let $A, \omega_{1}, \ldots, \omega_{r}, x$ range over the domain

$$
0<A<\min _{1 \leq i \leq r} \min \left(\Im\left(-x / \omega_{i}\right), \Im\left((x-1) / \omega_{i}\right)\right) .
$$

Then as $v \rightarrow 0^{+}$, we have the estimate

$$
-2 \pi \sqrt{-1} Q^{(r)}\left(x ; v \omega_{1}, \ldots, v \omega_{r}\right)+\log \Gamma_{e}^{(r)}\left(e(x) ; e\left(v \omega_{1}\right), \ldots, e\left(v \omega_{r}\right)\right)=O(\exp (-2 \pi A / v)),
$$

uniform over compact subsets of the domain. 
Proof. Using the "product" expansion and the identity

$$
\log \Gamma_{h}^{(r)}\left(z ; v \omega_{1}, \ldots, v \omega_{r}\right)=\log \Gamma_{h}^{(r)}\left(z / v ; \omega_{1}, \ldots, \omega_{r}\right),
$$

we can express each left-hand side as a sum over functions to which Corollary 2.3 applies, giving the desired uniform asymptotics.

Using this limit, we can obtain the following bound.

Proposition 2.12. Fix a compact subset $S$ of the set of $\omega_{i}$ such that $0<\Im\left(\omega_{i}\right)$, and constants $0<\epsilon, C_{1}, C_{2}$. Then as $v \rightarrow 0^{+}$, we have the following estimate, uniform over the product of $S$ with the region $-1-v C_{1} \leq$ $\Re(x) \leq v C_{1},|\Im(x)| \leq v C_{2}$, apart from a hole of radius $\epsilon v$ around every pole of the left-hand side:

$$
\Gamma_{e}^{(r)}\left(e(x) ; e\left(v \omega_{1}\right), \ldots, e\left(v \omega_{r}\right)\right)^{ \pm 1}=O\left(e\left( \pm Q^{(r)}\left(x ; v \omega_{1}, \ldots, v \omega_{r}\right)\right)\right) .
$$

Proof. We consider the + case; the - case is completely analogous. Choose $1 / 2<D<1$ and $0<a<$ $\min _{i} \Im\left(-1 / \omega_{i}\right)$. Proposition 2.10 gives

$$
e\left(-Q^{(r)}\left(x ; v \omega_{1}, \ldots, v \omega_{r}\right)\right) \Gamma_{e}^{(r)}\left(e(x) ; e\left(v \omega_{1}\right), \ldots, e\left(v \omega_{r}\right)\right)=O\left(e\left(\frac{1}{2} P^{(r)}\left(x ; \omega_{1}, \ldots, \omega_{r}\right)\right) \Gamma_{h}^{(r)}\left(x ; v \omega_{1}, \ldots, v \omega_{r}\right)\right)
$$

away from the poles, in the subregion $-D \leq \Re(x) \leq v C_{1}$. Since

$$
e\left(\frac{1}{2} P^{(r)}\left(x ; v \omega_{1}, \ldots, v \omega_{r}\right)\right) \Gamma_{h}^{(r)}\left(x ; v \omega_{1}, \ldots, v \omega_{r}\right)
$$

is uniformly bounded in that region (we have excluded neighborhoods of the poles, and it converges uniformly to 1 for $-\Re(x) / v$ large), we have the uniform estimate

$$
e\left(-Q^{(r)}\left(x ; v \omega_{1}, \ldots, v \omega_{r}\right)\right) \Gamma_{e}^{(r)}\left(e(x) ; e\left(v \omega_{1}\right), \ldots, e\left(v \omega_{r}\right)\right)=O(1)
$$

in this region. A similar application of Proposition 2.10 gives

$$
\begin{aligned}
e\left(-Q^{(r)}\left(x+1 ; v \omega_{1}, \ldots, v \omega_{r}\right)\right) & \Gamma_{e}^{(r)}\left(e(x) ; e\left(v \omega_{1}\right), \ldots, e\left(v \omega_{r}\right)\right) \\
= & O\left(e\left(\frac{1}{2} P^{(r)}\left(x+1 ; v \omega_{1}, \ldots, v \omega_{r}\right)\right) \Gamma_{h}^{(r)}\left(x+1 ; v \omega_{1}, \ldots, v \omega_{r}\right)\right)
\end{aligned}
$$

uniformly over the subset $-v C_{1} \leq \Re(x)+1 \leq D$. Since

$$
Q^{(r)}\left(x+1 ; v \omega_{1}, \ldots, v \omega_{r}\right)=Q^{(r)}\left(x ; v \omega_{1}, \ldots, v \omega_{r}\right)-P^{(r)}\left(x+1 ; v \omega_{1}, \ldots, v \omega_{r}\right),
$$

we find

$$
\begin{aligned}
e\left(-Q^{(r)}\left(x ; v \omega_{1}, \ldots, v \omega_{r}\right)\right) & \Gamma_{e}^{(r)}\left(e(x) ; e\left(v \omega_{1}\right), \ldots, e\left(v \omega_{r}\right)\right) \\
& =O\left(e\left(-\frac{1}{2} P^{(r)}\left(x+1 ; v \omega_{1}, \ldots, v \omega_{r}\right)\right) \Gamma_{h}^{(r)}\left(x+1 ; v \omega_{1}, \ldots, v \omega_{r}\right)\right)=O(1)
\end{aligned}
$$

on this region as well.

Similarly, we can obtain asymptotics of $\Gamma_{e}^{(r)}$ in the "rational" limit $p_{r} \rightarrow 1, p_{1}, \ldots p_{r-1}$ fixed. 
Theorem 2.13. Let $x, p_{1}, \ldots, p_{r-1}, \omega_{r}, \alpha, \beta$ range over the domain $0<\left|p_{1}\right|, \ldots,\left|p_{r-1}\right|<1, \Im\left(\omega_{r}\right)>0$, and

$$
x \in e^{-1}\left(\mathcal{C}_{e}^{(r-1)}\left(\omega_{r} ; p_{1}, \ldots, p_{r-1}\right)\right) \cup\left(\omega_{r} \mathbb{R}_{\geq 0} \cap e^{-1}\left(\mathcal{C}_{e}^{\prime(r-1)}\left(\omega_{r} ; p_{1}, \ldots, p_{r-1}\right)\right) .\right.
$$

Then as $v \rightarrow 0^{+}$, we have the estimate

$$
\begin{aligned}
\log \frac{\Gamma_{e}^{(r)}\left(e\left(x+v \alpha \omega_{r}\right) ; p_{1}, \ldots, p_{r-1}, e\left(v \omega_{r}\right)\right)}{\Gamma_{e}^{(r)}\left(e\left(x+v \beta \omega_{r}\right) ; p_{1}, \ldots, p_{r-1}, e\left(v \omega_{r}\right)\right)}-(-1)^{r} \log \frac{\Gamma_{r}\left(x+v \alpha \omega_{r} ; v \omega_{r}\right)}{\Gamma_{r}\left(x+v \beta \omega_{r} ; v \omega_{r}\right)} \\
-(\alpha-\beta)\left(\log \Gamma_{e}^{(r-1)}\left(e(x) ; p_{1}, \ldots, p_{r-1}\right)-(-1)^{r} \log \left(x / v \omega_{r}\right)\right)=O(v),
\end{aligned}
$$

uniformly over compact subsets of the domain.

Proof. If we expand the elliptic gamma functions via the product representation and group corresponding terms, we find by Corollary 2.5 that all but one term of the result is uniformly $O(v)$. Moreover, it follows from Theorem 2.4 that the coefficient of $v$ in the estimates is exponentially small as $k \rightarrow \infty$, and thus the error terms are summable. The only surviving term can be estimated using Theorem 2.8, giving the desired result.

Corollary 2.14. As $v \rightarrow 0^{+}$,

$$
\log \frac{\Gamma_{e}^{(2)}(e(\alpha v \omega) ; p, e(v \omega))}{\Gamma_{r}(\alpha)}=\frac{\pi \sqrt{-1}}{12 v \omega}+(\alpha-1 / 2) \log \left(2 \pi v \omega(p ; p)^{2} / \sqrt{-1}\right)+O(v)
$$

uniformly over compact subsets of the region $\Im(\omega)>0,0<|p|<1, \alpha \in \mathbb{C}$.

Similarly, one has the following.

Corollary 2.15. As $v \rightarrow 0^{+}$,

$$
\frac{\Gamma_{e}^{(r)}\left(e(\alpha v \omega) z ; p_{1}, \ldots, p_{r-1}, e(v \omega)\right)}{\Gamma_{e}^{(r)}\left(e(\beta v \omega) z ; p_{1}, \ldots, p_{r-1}, e(v \omega)\right)}=\Gamma_{e}^{(r-1)}\left(z ; p_{1}, \ldots, p_{r-1}\right)^{\alpha-\beta}(1+O(v))
$$

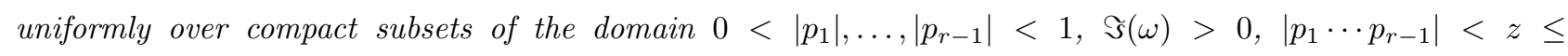
$\min _{1 \leq i \leq r-1}\left|p_{i}\right|^{-1}, z \neq 1, \alpha, \beta \in \mathbb{C}$.

Corollary 2.16. As $v \rightarrow 0^{+}$, the function

$$
\frac{\Gamma_{e}^{(r)}\left(e(v \alpha \omega) z ; p_{1}, \ldots, p_{r}, e(v \omega)\right)}{\Gamma_{e}^{(r)}\left(e(v \beta \omega) z ; p_{1}, \ldots, p_{r}, e(v \omega)\right)}
$$

is uniformly bounded over compact subsets of the domain $0<\left|p_{1}\right|, \ldots,\left|p_{r-1}\right|<1, \Im(\omega)>0,\left|p_{1} \cdots p_{r-1}\right|<z<$ $1, \alpha, \beta \in \mathbb{C}$. On compact subsets of the domain $0<\left|p_{1}\right|, \ldots,\left|p_{r-1}\right|<1, \Im(\omega)>0,|p|<z \leq 1, \alpha, \beta \in \mathbb{C}$, it is uniformly

$$
O\left(v^{\min (\Re(\alpha-\beta), 0)}\right) .
$$

There are corresponding estimates for the trigonometric gamma function

$$
\Gamma_{t}(z ; q):=\prod_{0 \leq k}\left(1-q^{k} z\right)^{-1}=\frac{1}{(z ; q)_{\infty}},
$$

with the corresponding analytic continuation of its logarithm (the branch with $\left.\log \Gamma_{t}(0 ; q)=0\right)$. 
Lemma 2.17. [10] Let $z, \omega, \alpha, \beta$ range over the domain $\Im(\omega)>0,0 \leq|z|<1$. Then as $v \rightarrow 0$, we have the estimate

$$
\log \frac{\Gamma_{t}(e(v \alpha \omega) z ; e(v \omega))}{\Gamma_{t}(e(v \beta \omega) z ; e(v \omega))}-(\alpha-\beta) \log (1-z)=O(v z)
$$

uniformly over compact subsets of the domain.

Proof. Indeed, for $|z|<1$, the left-hand side is given by the sum

$$
\sum_{k \geq 1} \frac{z^{k}}{k}\left(\frac{e(k v \alpha \omega)-e(k v \beta \omega)}{1-e(k v \omega)}+\alpha-\beta\right)
$$

and the quantity in parentheses is uniformly $O(v \exp (\epsilon k))$ for all $\epsilon>0$.

This gives rise to a trigonometric analogue of Theorem 2.13.

Theorem 2.18. For $r>1$, let $z, p_{1}, \ldots, p_{r-1}, \omega, \alpha, \beta$ range over the domain $0 \leq\left|p_{1}\right|, \ldots,\left|p_{r-1}\right|<1, \Im(\omega)>0$, and $z \in \mathcal{C}_{e}^{\prime(r-1)}\left(\omega ; p_{1}, \ldots, p_{r-1}\right)$. Then as $v \rightarrow 0^{+}$, we have the estimate

$$
\begin{gathered}
\log \frac{\Gamma_{e}^{(r)}\left(e(v \alpha \omega) z ; p_{1}, \ldots, p_{r-1}, e(v \omega)\right)}{\Gamma_{e}^{(r)}\left(e(v \beta \omega) z ; p_{1}, \ldots, p_{r-1}, e(v \omega)\right)}-(-1)^{r} \log \frac{\Gamma_{t}(e(v \alpha \omega) z ; e(v \omega))}{\Gamma_{t}(e(v \beta \omega) z ; e(v \omega))} \\
-(\alpha-\beta)\left(\log \Gamma_{e}^{(r-1)}\left(z ; p_{1}, \ldots, p_{r-1}\right)-(-1)^{r} \log (1-z)\right)=O(v),
\end{gathered}
$$

uniformly over compact subsets of the domain.

Proof. For $\left|p_{1} p_{2} \cdots p_{r-1}\right|<z<\min _{i}\left|p_{i}\right|^{-1}$, this follows immediately from the expansion

$$
\log \Gamma_{e}^{(r)}\left(z ; p_{1}, \ldots, p_{r-1}, q\right)=-\sum_{0 \leq k_{1}, k_{2}, \ldots, k_{r-1}} \Gamma_{t}\left(p_{1}^{k_{1}+1} \cdots p_{r-1}^{k_{r-1}+1} / z ; q\right)+(-1)^{r-1} \Gamma_{t}\left(p_{1}^{k_{1}} \cdots p_{r-1}^{k_{r-1}} z ; q\right)
$$

and the asymptotics of $\Gamma_{t}$.

The general case follows from the functional equation.

Comparing this to Theorem 2.13 gives the following result, a uniform version of the results of the appendices of [10].

Corollary 2.19. Let $x, \omega, \alpha, \beta$ range over the domain $\Im(\omega)>0$,

$$
x \in\left(\mathbb{C} \backslash\left(\mathbb{Z}+\omega \mathbb{R}_{\geq 0}\right)\right) \cup \omega \mathbb{R}_{\geq 0} .
$$

Then as $v \rightarrow 0^{+}$, we have the estimate

$$
\log \frac{\Gamma_{t}(e(x+v \alpha \omega) ; e(v \omega))}{\Gamma_{t}(e(x+v \beta \omega) ; e(v \omega))}-\log \frac{\Gamma_{r}(x+v \alpha \omega ; v \omega)}{\Gamma_{r}(x+v \beta \omega ; v \omega)}-(\alpha-\beta)(\log (1-e(x))-\log (x / v \omega))=O(v),
$$

uniformly over compact subsets of the domain.

Remark. Note that the validity of the theorem for $r=2,|p|<z<|p|^{-1}$ is enough to give the corollary in general, and in turn give the theorem in general, without using the functional equation. This also implies that Theorem 2.13 and its corollaries continue to hold even without the constraint $0<\left|p_{i}\right|$ on the domain, and further implies that Lemma 2.17 holds on the domain $z \notin e\left(\omega \mathbb{R}_{\geq 0}\right)$. 
Corollary 2.20. [10] Let $\omega$, $\alpha$ range over the domain $\Im(\omega)>0, \alpha \in \mathbb{C}$. Then as $v \rightarrow 0^{+}$, we have the estimate

$$
\log \frac{\Gamma_{t}(e(v \alpha \omega) ; e(v \omega))}{\Gamma_{r}(\alpha)}=\frac{\pi \sqrt{-1}}{12 v \omega}+(\alpha-1 / 2) \log (2 \pi v \omega / \sqrt{-1})+O(v),
$$

uniformly over compact subsets of the domain.

\section{Generalized triangle inequalities}

When taking limits of elliptic hypergeometric integrals, the first step is naturally to determine which part of the contour makes the most significant contribution to the integral. We first note the following consequence of Proposition 2.12 Since in the sequel, we will only be using gamma functions for $r \leq 3$, we will write $\theta$ for $\Gamma_{e}^{(1)}$, $\Gamma_{e}$ for $\Gamma_{e}^{(2)}$, and $\Gamma_{e}^{+}$for $\Gamma_{e}^{(3)}$, and similarly (with a subscript $h$ ) for the hyperbolic versions; we will also omit the superscript $(r)$ on $P, Q$, and $R$.

Corollary 3.1. For any parameters $\mu, \nu$, and any real number $x$, we have, as $v \rightarrow 0^{+}$, the estimates (uniform over compact subsets avoiding the poles)

$$
\begin{aligned}
\frac{\Gamma_{e}\left(e(v \mu+x), e(v \nu-x) ; e\left(v \omega_{1}\right), e\left(v \omega_{2}\right)\right)}{e\left(R\left(v \mu ; v \omega_{1}, v \omega_{2}\right)+R\left(v \nu ; v \omega_{1}, v \omega_{2}\right)\right)} & =O\left(e\left(\frac{\mu+\nu-\omega_{1}-\omega_{2}}{2 v \omega_{1} \omega_{2}} \vartheta(x)\right)\right), \\
\frac{e\left(R\left(v \nu ; v \omega_{1}, v \omega_{2}\right)\right) \Gamma_{e}\left(e(v \mu+x) ; e\left(v \omega_{1}\right), e\left(v \omega_{2}\right)\right)}{e\left(R\left(v \mu ; v \omega_{1}, v \omega_{2}\right)\right) \Gamma_{e}\left(e(v \nu+x) ; e\left(v \omega_{1}\right), e\left(v \omega_{2}\right)\right)} & =O\left(e\left(\frac{\mu-\nu}{2 v \omega_{1} \omega_{2}} \vartheta(x)\right)\right), \\
\frac{e\left(R\left(v \mu ; v \omega_{1}, v \omega_{2}\right)+R\left(v \nu ; v \omega_{1}, v \omega_{2}\right)\right)}{\Gamma_{e}\left(e(v \mu+x), e(v \nu-x) ; e\left(v \omega_{1}\right), e\left(v \omega_{2}\right)\right)} & =O\left(e\left(\frac{\omega_{1}+\omega_{2}-\mu-\nu}{2 v \omega_{1} \omega_{2}} \vartheta(x)\right)\right),
\end{aligned}
$$

where $\vartheta(x)$ is the continuous, even, periodic function defined by

$$
\vartheta(x)=\{x\}(1-\{x\})=\{x\}\{-x\} .
$$

Similarly,

$$
e(-R(v \mu ; v \omega)) \theta(e(v \mu+x) ; e(v \omega))=O(e(\vartheta(x) / 2 v \omega))
$$

and likewise for the reciprocal (avoiding the poles).

Remark. Note that in the above bounds, we can ignore $O(1)$ terms in $R$, and may thus replace

$$
\begin{aligned}
R(v \mu ; v \omega) & \mapsto-1 / 12 v \omega \\
R\left(v \mu ; v \omega_{1}, v \omega_{2}\right) & \mapsto\left(\omega_{1}+\omega_{2}-2 \mu\right) / 24 v \omega_{1} \omega_{2} .
\end{aligned}
$$

Also, although we assume $x$ real, the above estimates are clearly still valid if we make a $O(v)$ perturbation to $x$ on the left-hand sides.

We will thus require some inequalities involving this quantity $\vartheta$.

Lemma 3.2. For any sequences $c_{1}, \ldots, c_{n}, d_{1}, \ldots, d_{n}$ of real numbers, we have the inequality

$$
\sum_{1 \leq i, j \leq n} \vartheta\left(c_{i}-d_{j}\right)-\sum_{1 \leq i<j \leq n} \vartheta\left(c_{i}-c_{j}\right)-\sum_{1 \leq i<j \leq n} \vartheta\left(d_{i}-d_{j}\right) \geq \vartheta\left(\sum_{1 \leq i \leq n} c_{i}-d_{i}\right),
$$


with equality if and only if the sequences interlace in $\mathbb{R} / \mathbb{Z}$; that is, iff they can be permuted so that either

$$
\left\{c_{1}\right\} \leq\left\{d_{1}\right\} \leq\left\{c_{2}\right\} \leq \cdots \leq\left\{d_{n-1}\right\} \leq\left\{c_{n}\right\} \leq\left\{d_{n}\right\}
$$

or

$$
\left\{d_{1}\right\} \leq\left\{c_{1}\right\} \leq\left\{d_{2}\right\} \leq \cdots \leq\left\{c_{n-1}\right\} \leq\left\{d_{n}\right\} \leq\left\{c_{n}\right\} .
$$

Proof. First, observe that if two elements $c_{i}, d_{j}$ agree modulo $\mathbb{Z}$, then their contributions to the inequality cancel, and the result thus follows by induction. We may therefore assume that $c_{i} \neq d_{j}$ for all $1 \leq i, j \leq n$.

Now, consider the asymptotics as $v \rightarrow 0^{+}$of the case $\tau=\omega / 2$ of the determinant identity [7]:

$$
\begin{aligned}
\operatorname{det}_{1 \leq i, j \leq n}\left(\frac{e(-1 / 12 v \omega) \theta\left(e\left(v \tau+c_{i}^{\prime}-d_{j}^{\prime}\right) ; e(v \omega)\right)}{\theta\left(e(v \tau), e\left(c_{i}^{\prime}-d_{j}^{\prime}\right) ; e(v \omega)\right)}\right) & \\
= & (-1)^{n(n-1) / 2} \frac{\theta\left(e\left(v \tau+\sum_{i} c_{i}^{\prime}-d_{i}^{\prime}\right) ; e(v \omega)\right)}{e(n / 12 v \omega) \theta(e(v \tau) ; e(v \omega))} \frac{\prod_{1 \leq i<j \leq n} e\left(c_{j}^{\prime}-d_{i}^{\prime}\right) \theta\left(e\left(c_{i}^{\prime}-c_{j}^{\prime}\right), e\left(d_{i}^{\prime}-d_{j}^{\prime}\right) ; e(v \omega)\right)}{\prod_{1 \leq i, j \leq n} \theta\left(e\left(c_{i}^{\prime}-d_{j}^{\prime}\right) ; e(v \omega)\right)},
\end{aligned}
$$

where real constants $\left(c_{i}^{\prime}-c_{i}\right) / v,\left(d_{i}^{\prime}-d_{i}\right) / v$ are chosen so that the $2 n$ quantities $c_{i}^{\prime}, d_{i}^{\prime}$ are all distinct for sufficiently small $v$. Now, since $c_{i} \neq d_{j}$, we have

$$
\lim _{v \rightarrow 0^{+}} \frac{e(-1 / 12 v \omega) \theta\left(e\left(v \omega / 2+c_{i}^{\prime}-d_{j}^{\prime}\right) ; e(v \omega)\right)}{\theta\left(e(v \omega / 2), e\left(c_{i}^{\prime}-d_{j}^{\prime}\right) ; e(v \omega)\right)}=\frac{1}{2} \operatorname{sgn}\left(\left\{c_{i}\right\}-\left\{d_{j}\right\}\right) e\left(-\left(\left\{c_{i}\right\}-\left\{d_{j}\right\}\right) / 2+1 / 4\right) .
$$

In particular, the determinant converges to a well-defined limit. Moreover, this limit is nonzero iff the sequences interlace, as follows by considering the rescaled determinant $\operatorname{det}_{1 \leq i, j \leq n}\left(\operatorname{sgn}\left(\left\{c_{i}\right\}-\left\{d_{j}\right\}\right)\right)$. (Indeed, if the sequences fail to interlace, two rows or columns must agree; otherwise, the $n$ distinct rows are easily verified to be linearly independent.)

On the other hand, we have the estimates

$$
\begin{aligned}
\frac{\theta(e(v \omega / 2+x) ; e(v \omega))}{\theta(e(v \omega / 2) ; e(v \omega))} & =\Theta(e(\vartheta(x) / 2 v \omega)) \\
e(1 / 12 v \omega) \theta(e(x) ; e(v \omega)) & =\Theta(e(\vartheta(x) / 2 v \omega)) \quad \text { assuming }|\{x\}|,|\{1-x\}|=\Omega(v),
\end{aligned}
$$

giving the estimate

$$
\Theta\left(e\left(\left(\vartheta\left(\sum_{i} c_{i}-d_{i}\right)+\sum_{1 \leq i<j \leq n}\left(\vartheta\left(c_{i}-c_{j}\right)+\vartheta\left(d_{i}-d_{j}\right)\right)-\sum_{1 \leq i, j \leq n} \vartheta\left(c_{i}-d_{j}\right)\right) / 2 v \omega\right)\right)
$$

for the right-hand side. Since $\Im(-1 / \omega)>0$, this is bounded as $v \rightarrow 0^{+}$iff

$$
\vartheta\left(\sum_{i} c_{i}-d_{i}\right)+\sum_{1 \leq i<j \leq n}\left(\vartheta\left(c_{i}-c_{j}\right)+\vartheta\left(d_{i}-d_{j}\right)\right)-\sum_{1 \leq i, j \leq n} \vartheta\left(c_{i}-d_{j}\right) \leq 0,
$$

and is bounded away from 0 iff equality holds. The result follows.

Remark. More precise asymptotic calculations give the following well-known (and easy) identity (valid for $n \geq 1$ ) as a limit of the elliptic Cauchy determinant:

$$
\operatorname{det}_{1 \leq i, j \leq n}\left(\operatorname{sgn}\left(x_{i}-y_{j}\right)\right)=2^{n-1}(-1)^{n(n-1)} \prod_{1 \leq i, j \leq n} \operatorname{sgn}\left(x_{i}-y_{j}\right) \prod_{1 \leq i<j \leq n} \operatorname{sgn}\left(x_{i}-x_{j}\right) \operatorname{sgn}\left(y_{i}-y_{j}\right)
$$


for interlacing sequences with distinct elements, and 0 otherwise. Similarly, for more general values of $\tau$, we obtain the identity

$$
\operatorname{det}_{1 \leq i, j \leq n}\left(x^{\operatorname{sgn}\left(c_{i}-d_{j}\right)}\right)=(-1)^{n(n-1) / 2}(x-1 / x)^{n-1} \operatorname{sgn}\left(\sum_{i} c_{i}-d_{i}\right) x^{\operatorname{sgn}\left(\sum_{i} c_{i}-d_{i}\right)} \frac{\prod_{1 \leq i<j \leq n} \operatorname{sgn}\left(c_{i}-c_{j}\right) \operatorname{sgn}\left(d_{i}-d_{j}\right)}{\prod_{1 \leq i, j \leq n} \operatorname{sgn}\left(c_{i}-d_{j}\right)} .
$$

We can also obtain a version with hyperoctahedral symmetry.

Lemma 3.3. For any sequences $c_{0}, \ldots, c_{n}, d_{1}, \ldots, d_{n}$ of real numbers, we have the inequality

$$
\sum_{0 \leq i \leq n, 1 \leq j \leq n} \vartheta\left(c_{i} \pm d_{j}\right)-\sum_{0 \leq i<j \leq n} \vartheta\left(c_{i} \pm c_{j}\right)-\sum_{1 \leq i<j \leq n} \vartheta\left(d_{i} \pm d_{j}\right)-\sum_{1 \leq i \leq n} \vartheta\left(2 d_{i}\right) \geq 0
$$

with equality iff the sequences can be permuted so that

$$
\min \left(\left\{ \pm c_{0}\right\}\right) \leq \min \left(\left\{ \pm d_{1}\right\}\right) \leq \min \left(\left\{ \pm c_{1}\right\}\right) \leq \cdots \leq \min \left(\left\{ \pm c_{n-1}\right\}\right) \leq \min \left(\left\{ \pm d_{n}\right\}\right) \leq \min \left(\left\{ \pm c_{n}\right\}\right)
$$

Here $\vartheta(x \pm y):=\vartheta(x+y)+\vartheta(x-y)$ and $\min (\{ \pm x\}):=\min (\{x\},\{-x\})$.

Proof. Apply the preceding lemma to the sequences $\pm c_{i}$ and $0, \pm d_{i}, 1 / 2$, and use the identity

$$
\vartheta(2 x)=2(\vartheta(x)+\vartheta(x+1 / 2)-\vartheta(1 / 2)) .
$$

The given equality condition simply restates the condition that $\pm c_{i}$ and $0, \pm d_{i}, 1 / 2$ interlace.

Corollary 3.4. For any integer $n \geq 1$ and any sequence $e_{1}, \cdots, e_{n}$ of real numbers, we have the inequality

$$
2 \sum_{1 \leq i<j \leq n} \vartheta\left(e_{i} \pm e_{j}\right)-(n-1) \sum_{1 \leq i \leq n} \vartheta\left(2 e_{i}\right) \geq 0
$$

with equality iff the sequence $\min \left(\left\{ \pm e_{i}\right\}\right)$ is constant.

Proof. The case $c_{0}=0, n=1$ of Lemma 3.3 implies that

$$
\vartheta(x \pm y)+2(\vartheta(x)-\vartheta(y))-\vartheta(2 x) \geq 0
$$

with equality iff $\min (\{ \pm x\}) \leq \min (\{ \pm y\})$. Adding all specializations of the form $(x, y) \mapsto\left(e_{i}, e_{j}\right)$ with $i \neq j$ gives the desired result.

If we rescale $c_{i}, d_{i} \mapsto v c_{i}, v d_{i}$ and take $v \rightarrow 0^{+}$, the fact that $\vartheta(v x)=v|x|-v^{2} x^{2}$ for sufficiently small $v$ gives us the following limit.

Lemma 3.5. For any sequences $c_{1}, \ldots, c_{n}, d_{1}, \ldots, d_{n}$, of real numbers, we have the following inequality:

$$
\sum_{\substack{1 \leq i \leq n \\ 1 \leq j \leq n}}\left|c_{i}-d_{j}\right|-\sum_{1 \leq i<j \leq n}\left|c_{i}-c_{j}\right|-\sum_{1 \leq i<j \leq n}\left|d_{i}-d_{j}\right| \geq\left|\sum_{1 \leq i \leq n} c_{i}-\sum_{1 \leq i \leq n} d_{i}\right|,
$$

with equality iff the sequences can be permuted so that

$$
c_{1} \leq d_{1} \leq \cdots \leq c_{n} \leq d_{n}
$$

or

$$
d_{1} \leq c_{1} \leq \cdots \leq d_{n} \leq c_{n} .
$$


In particular, we have the following fact.

Lemma 3.6. For any sequences $c_{0}, \ldots, c_{n}, d_{1}, \ldots, d_{n}$, of real numbers, we have the following inequality:

$$
\sum_{\substack{0 \leq i \leq n \\ 1 \leq j \leq n}}\left|c_{i}-d_{j}\right|-\sum_{0 \leq i<j \leq n}\left|c_{i}-c_{j}\right|-\sum_{1 \leq i<j \leq n}\left|d_{i}-d_{j}\right| \geq 0,
$$

with equality iff the sequences can be permuted so that

$$
c_{0} \leq d_{1} \leq c_{1} \leq \cdots \leq c_{n-1} \leq d_{n} \leq c_{n}
$$

Proof. Choose a number $d_{0}$ such that $d_{0}<\min \left(c_{0}, \ldots, c_{n}, d_{1}, \ldots, d_{n}, \sum_{i} c_{i}-\sum_{i>0} d_{i}\right)$, and apply Lemma 3.5 .

Remark. The case $n=1$ is of course just the usual triangle inequality in $\mathbb{R}$, thus justifying the title of this section.

\section{Hyperbolic limits}

Using the above asymptotic estimates for the hyperbolic and elliptic gamma functions, we can obtain corresponding estimates for the various elliptic hypergeometric integrals of [16] in the hyperbolic limit. In particular, in each case, it will turn out that up to an explicit exponential factor, the elliptic integral converges exponentially quickly to the hyperbolic integral.

Let us first consider the case of the Type I (perhaps better named "elliptic Dixon", see Corollary 7.3 below and [6]) integral with $B C_{n}$ symmetry, defined for all nonnegative integers $m, n$, and parameters $p, q$, $u_{0} \ldots u_{2 m+2 n+3}$ satisfying

$$
0<|p|,|q|<1, \quad \prod_{0 \leq r \leq 2 m+2 n+3} u_{r}=(p q)^{m+1}
$$

by the integral

$$
I_{B C_{n}}^{(m)}\left(u_{0}, u_{1}, \ldots ; p, q\right):=\frac{(p ; p)^{n}(q ; q)^{n}}{2^{n} n !} \int_{C^{n}} \frac{\prod_{1 \leq i \leq n} \prod_{0 \leq r \leq 2 m+2 n+3} \Gamma_{e}\left(u_{r} z_{i}^{ \pm 1} ; p, q\right)}{\prod_{1 \leq i<j \leq n} \Gamma_{e}\left(z_{i}^{ \pm 1} z_{j}^{ \pm 1} ; p, q\right) \prod_{1 \leq i \leq n} \Gamma_{e}\left(z_{i}^{ \pm 2} ; p, q\right)} \prod_{1 \leq i \leq n} \frac{d z_{i}}{2 \pi \sqrt{-1} z_{i}},
$$

where the contour is chosen to contain all points of the form $p^{i} q^{j} u_{r}, 0 \leq i, j$, and exclude their reciprocals.

In the hyperbolic limit $p, q, u_{r} \rightarrow 1$, this gives rise to the following limit.

Theorem 4.1. Let $\mu_{0}, \mu_{1}, \ldots, \mu_{2 m+2 n+3}, \omega_{1}, \omega_{2}$ be parameters such that

$$
\Im\left(\omega_{1}\right), \Im\left(\omega_{2}\right)>0, \quad \sum_{r} \mu_{r}=(m+1)\left(\omega_{1}+\omega_{2}\right) .
$$

Then as $v \rightarrow 0^{+}$,

$$
e\left(-2 n \sum_{r} R\left(v \mu_{r} ; v \omega_{1}, v \omega_{2}\right)+\left(2 n^{2}+n\right) R\left(0 ; v \omega_{1}, v \omega_{2}\right)\right) I_{B C_{n}}^{(m)}\left(e\left(v \mu_{0}\right), e\left(v \mu_{1}\right), \ldots ; e\left(v \omega_{1}\right), e\left(v \omega_{2}\right)\right)
$$


converges uniformly exponentially (over compact subsets) to

$$
\frac{1}{\left(\sqrt{-\omega_{1} \omega_{2}}\right)^{n} 2^{n} n !} \int_{C^{n}} \frac{\prod_{1 \leq i \leq n} \prod_{0 \leq r \leq 2 m+2 n+3} \Gamma_{h}\left(\mu_{r} \pm x_{i} ; \omega_{1}, \omega_{2}\right)}{\prod_{1 \leq i<j \leq n} \Gamma_{h}\left( \pm x_{i} \pm x_{j} ; \omega_{1}, \omega_{2}\right) \prod_{1 \leq i \leq n} \Gamma_{h}\left( \pm 2 x_{i} ; \omega_{1}, \omega_{2}\right)} \prod_{1 \leq i \leq n} d x_{i}
$$

where the contour agrees with $\mathbb{R}$ outside a compact set, and is chosen to contain all points of the form $\mu_{r}+$ $i \omega_{1}+j \omega_{2}, i, j \geq 0$ and exclude their negatives.

Proof. We first observe that

$$
\begin{aligned}
e\left(R\left(0 ; v \omega_{1}, v \omega_{2}\right)\right)\left(e\left(v \omega_{1}\right) ; e\left(v \omega_{1}\right)\right)\left(e\left(v \omega_{2}\right) ; e\left(v \omega_{2}\right)\right) & =\lim _{z \rightarrow 0} \frac{e\left(R\left(v z ; v \omega_{1}, v \omega_{2}\right)\right)}{(1-e(v z)) \Gamma_{e}\left(e(v z) ; e\left(v \omega_{1}\right), e\left(v \omega_{2}\right)\right)} \\
& \sim \lim _{z \rightarrow 0} \frac{1}{(1-e(v z)) \Gamma_{h}\left(z ; \omega_{1}, \omega_{2}\right)} \\
& =\frac{1}{v \sqrt{-\omega_{1} \omega_{2}}},
\end{aligned}
$$

with uniform exponentially small relative error as $v \rightarrow 0^{+}$.

For the remaining factors, we first assume that $\Im\left(\mu_{r}\right)>0$ for all $r$, and thus the elliptic contour may be taken to be the unit circle. Now, in the elliptic integral, introduce the change of variables $z_{i}=e\left(x_{i}\right)$, and thus $d z_{i} / 2 \pi \sqrt{-1} z_{i}=d x_{i}$; this replaces the unit circle by the cube $[-1 / 2,1 / 2]^{n}$. We next claim that if we restrict to the smaller cube $[-1 / 4,1 / 4]^{n}$, the resulting error is uniformly exponentially small. Indeed, we can use Corollary 3.1 to bound the integrand on the full cube. The $\mu$ factors satisfy

$$
e\left(-2 R\left(v \mu_{r} ; v \omega_{1}, v \omega_{2}\right)\right) \Gamma_{e}\left(e\left(v \mu_{r} \pm x_{i}\right) ; e\left(v \omega_{1}\right), e\left(v \omega_{2}\right)\right)=O\left(e\left(\frac{-\omega_{1}-\omega_{2}+2 \mu_{r}}{2 v \omega_{1} \omega_{2}} \vartheta\left(x_{i}\right)\right)\right)
$$

and thus, using the balancing condition,

$$
\prod_{0 \leq r \leq 2 m+2 n+3} e\left(-2 R\left(v \mu_{r} ; v \omega_{1}, v \omega_{2}\right)\right) \Gamma_{e}\left(e\left(v \mu_{r} \pm x_{i}\right) ; e\left(v \omega_{1}\right), e\left(v \omega_{2}\right)\right)=O\left(e\left(\frac{-\omega_{1}-\omega_{2}}{2 v \omega_{1} \omega_{2}} 2(n+1) \vartheta\left(x_{i}\right)\right)\right) .
$$

Similarly, the remaining univariate factors satisfy

$$
e\left(2 R\left(0 ; v \omega_{1}, v \omega_{2}\right)\right) \Gamma_{e}\left(e\left( \pm 2 x_{i}\right) ; e\left(v \omega_{1}\right), e\left(v \omega_{2}\right)\right)^{-1}=O\left(e\left(\frac{-\omega_{1}-\omega_{2}}{2 v \omega_{1} \omega_{2}}\left(-\vartheta\left(2 x_{i}\right)\right)\right)\right)
$$

and, for $i<j$, the cross factors satisfy

$$
e\left(4 R\left(0 ; v \omega_{1}, v \omega_{2}\right)\right) \Gamma_{e}\left(e\left( \pm x_{i} \pm x_{j}\right) ; e\left(v \omega_{1}\right), e\left(v \omega_{2}\right)\right)^{-1}=O\left(e\left(\frac{-\omega_{1}-\omega_{2}}{2 v \omega_{1} \omega_{2}}\left(-\vartheta\left(x_{i} \pm x_{j}\right)\right)\right)\right)
$$

Combining these bounds, we find that the integrand is uniformly

$$
O\left(e\left(\frac{-\omega_{1}-\omega_{2}}{2 v \omega_{1} \omega_{2}}\left(\sum_{1 \leq i \leq n}(2 n+2) \vartheta\left(x_{i}\right)-\sum_{1 \leq i \leq n} \vartheta\left(2 x_{i}\right)-\sum_{1 \leq i<j \leq n} \vartheta\left(x_{i} \pm x_{j}\right)\right)\right)\right)
$$

Since

the bound is maximized when

$$
\Im\left(\frac{-\omega_{1}-\omega_{2}}{\omega_{1} \omega_{2}}\right)=\Im\left(-1 / \omega_{1}\right)+\Im\left(-1 / \omega_{2}\right)>0,
$$

$$
\sum_{1 \leq i \leq n}(2 n+2) \vartheta\left(x_{i}\right)-\sum_{1 \leq i \leq n} \vartheta\left(2 x_{i}\right)-\sum_{1 \leq i<j \leq n}\left(\vartheta\left(x_{i}+x_{j}\right)+\vartheta\left(x_{i}-x_{j}\right)\right)
$$


is minimized, which in turn happens when $x_{1}=x_{2}=\cdots=x_{n}=0$, by Lemma 3.3 applied to the case $c_{i} \equiv 0$, $d_{i}=x_{i}$. In particular, the integrand is exponentially small everywhere else, and thus restricting to $\left|x_{i}\right| \leq 1 / 4$ introduces an exponentially small error.

At this point, using Proposition 2.10 allows us to replace the gamma functions in the integrand with hyperbolic gamma functions (times an exponential factor that turns out to be trivial). The factor $v^{-n}$ from $((p ; p)(q ; q))^{n}$ can be absorbed in rescaling the variables of integration; we thus obtain the restriction of the desired integral to the cube $[-1 / 4 v, 1 / 4 v]$. But again we can bound the integrand, this time using Corollary 2.3. and find the uniform bound

$$
O\left(e\left(\frac{-\omega_{1}-\omega_{2}}{2 \omega_{1} \omega_{2}}\left(\sum_{1 \leq i \leq n}(2 n+2)\left|x_{i}\right|-\sum_{1 \leq i \leq n}\left|2 x_{i}\right|-\sum_{1 \leq i<j \leq n}\left(\left|x_{i}+x_{j}\right|+\left|x_{i}-x_{j}\right|\right)\right)\right)\right),
$$

so the omitted tail is again uniformly exponentially small.

For the general case, we note that if $C$ is a valid choice of contour for the hyperbolic integral, then for sufficiently small $v$, the image of the subcontour $[-1 / 2 v, 1 / 2 v]$ under $x \mapsto e(v x)$ is a valid choice of contour for the elliptic integral. The result agrees with the unit circle outside a neighborhood of size $O(v)$ of 1 ; as a result, the difference from the unit circle has no effect on the asymptotics.

If we denote the above integral by $I_{B C_{n} ; h}^{(m)}$, we have the following corollary, obtained as the limit of the corresponding identity for the elliptic case; note that we do not need to compare the exponential factors on both sides, since both sides must agree throughout and have generically nonzero limits.

Corollary 4.2. Let $\mu_{0}, \mu_{1}, \ldots, \mu_{2 m+2 n+3}, \omega_{1}, \omega_{2}$ be parameters such that

$$
\Im\left(\omega_{1}\right), \Im\left(\omega_{2}\right)>0, \quad \sum_{r} \mu_{r}=(m+1)\left(\omega_{1}+\omega_{2}\right) .
$$

Then

$$
I_{B C_{n} ; h}^{(m)}\left(\ldots, \mu_{r}, \ldots ; \omega_{1}, \omega_{2}\right)=\prod_{0 \leq r<s \leq 2 m+2 n+3} \Gamma_{h}\left(\mu_{r}+\mu_{s} ; \omega_{1}, \omega_{2}\right) I_{B C_{m} ; h}^{(n)}\left(\ldots, \frac{\omega_{1}+\omega_{2}}{2}-\mu_{r}, \ldots ; \omega_{1}, \omega_{2}\right),
$$

and in particular

$$
I_{B C_{n} ; h}^{(0)}\left(\ldots, \mu_{r}, \ldots ; \omega_{1}, \omega_{2}\right)=\prod_{0 \leq r<s \leq 2 n+3} \Gamma_{h}\left(\mu_{r}+\mu_{s} ; \omega_{1}, \omega_{2}\right) .
$$

Remark. As van Diejen and Spiridonov [5] observed for the Type II evaluation, one can also prove hyperbolic results by simply replacing the arguments of [16] by appropriate limits, rather than taking limits directly. Those arguments depend strongly on the fact that the set $p^{\mathbb{Z}} q^{\mathbb{Z}}$ generically has finite limit points (in fact, is generically dense), which makes analytic continuation trivial. In the hyperbolic setting, the corresponding set $\mathbb{Z} \omega_{1}+\mathbb{Z} \omega_{2}$ is never dense, and only has a limit point when $\omega_{1} / \omega_{2}$ is real irrational, so an additional analytic continuation argument is needed to extend to generic moduli.

A similar argument will work in the other cases; some technical issues do arise, however, so it is worth discussing those cases as well. 
For the Type II (again, the name "elliptic Selberg" might be better) integral, the main complication is that without an additional condition on the parameters, the integrand is not maximized near $z_{i} \equiv 1$. We have the following result. Define a family of integrals

$$
\begin{aligned}
& I I_{B C_{n}}^{(m)}\left(u_{0}, u_{1}, \ldots, u_{2 m+5} ; t ; p, q\right) \\
& \quad:=\frac{(p ; p)^{n}(q ; q)^{n} \Gamma_{e}(t ; p, q)^{n}}{2^{n} n !} \int_{C^{n}} \prod_{1 \leq i<j \leq n} \frac{\Gamma_{e}\left(t z_{i}^{ \pm 1} z_{j}^{ \pm 1} ; p, q\right)}{\Gamma_{e}\left(z_{i}^{ \pm 1} z_{j}^{ \pm 1} ; p, q\right)} \prod_{1 \leq i \leq n} \frac{\prod_{0 \leq r \leq 2 m+5} \Gamma_{e}\left(u_{r} z_{i}^{ \pm 1} ; p, q\right)}{\Gamma_{e}\left(z_{i}^{ \pm 2} ; p, q\right)} \frac{d z_{i}}{2 \pi \sqrt{-1} z_{i}},
\end{aligned}
$$

on the domain $t^{2 n-2} \prod_{r} u_{r}=(p q)^{m+1}, 0<|p|,|q|,|t|<1$, where the contour $C$ satisfies $C=C^{-1}$, and for all $i, j \geq 0$, contains the points $p^{i} q^{j} u_{r}$ as well as the contour $p^{i} q^{j} t C$.

Theorem 4.3. Let $\mu_{0}, \mu_{1}, \ldots, \mu_{2 m+5}, \tau, \omega_{1}, \omega_{2}$ be parameters such that

$$
\Im(\tau), \Im\left(\omega_{1}\right), \Im\left(\omega_{2}\right)>0, \quad(2 n-2) \tau+\sum_{r} \mu_{r}=(m+1)\left(\omega_{1}+\omega_{2}\right),
$$

and satisfying the convergence condition

$$
\Im\left(\frac{-(n-1) \tau-\omega_{1}-\omega_{2}}{\omega_{1} \omega_{2}}\right)>0 .
$$

Then as $v \rightarrow 0^{+}$,

$$
\begin{array}{r}
e\left(-2 n \sum_{r} R\left(v \mu_{r} ; v \omega_{1}, v \omega_{2}\right)+2 n^{2} R\left(0 ; v \omega_{1}, v \omega_{2}\right)-2 n(n-1) R\left(v \tau ; v \omega_{1}, v \omega_{2}\right)\right) \\
\cdot I I_{B C_{n}}^{(m)}\left(e\left(v \mu_{0}\right), e\left(v \mu_{1}\right), \ldots ; e(v \tau) ; e\left(v \omega_{1}\right), e\left(v \omega_{2}\right)\right)
\end{array}
$$

converges uniformly exponentially (over compact subsets) to

$$
\frac{\Gamma_{h}\left(\tau ; \omega_{1}, \omega_{2}\right)^{n}}{\left(\sqrt{-\omega_{1} \omega_{2}}\right)^{n} 2^{n} n !} \int_{C^{n}} \prod_{1 \leq i<j \leq n} \frac{\Gamma_{h}\left(\tau \pm x_{i} \pm x_{j} ; \omega_{1}, \omega_{2}\right)}{\Gamma_{h}\left( \pm x_{i} \pm x_{j} ; \omega_{1}, \omega_{2}\right)} \prod_{1 \leq i \leq n} \frac{\prod_{0 \leq r \leq 2 m+5} \Gamma_{h}\left(\mu_{r} \pm x_{i} ; \omega_{1}, \omega_{2}\right)}{\Gamma_{h}\left( \pm 2 x_{i} ; \omega_{1}, \omega_{2}\right)} d x_{i},
$$

where the contour $C=-C$ agrees with $\mathbb{R}$ outside a compact set and for all $i, j \geq 0$ contains the points $i \omega_{1}+j \omega_{2}+\mu_{r}$ as well as the contour $i \omega_{1}+j \omega_{2}+\tau+C$.

Proof. Again we change variables to $z_{i}=e\left(x_{i}\right)$ and integrate over the cube $[-1 / 2,1 / 2]^{n}$; we may also freely assume $n>1$, as the case $n=1$ has already been dealt with. In this case, we find that the integrand is uniformly bounded by

$$
\begin{aligned}
O & \left(e \left(\frac{-\omega_{1}-\omega_{2}}{2(n-1) v \omega_{1} \omega_{2}}\left(2 \sum_{1 \leq i<j \leq n} \vartheta\left(x_{i} \pm x_{j}\right)-(n-1) \sum_{1 \leq i \leq n} \vartheta\left(2 x_{i}\right)\right)\right.\right. \\
& \left.\left.+\frac{-(n-1) \tau-\omega_{1}-\omega_{2}}{(n-1) v \omega_{1} \omega_{2}}\left(2(n-1) \sum_{1 \leq i \leq n} \vartheta\left(x_{i}\right)-\sum_{1 \leq i<j \leq n} \vartheta\left(x_{i} \pm x_{j}\right)\right)\right)\right) .
\end{aligned}
$$

By Corollary [3.4 the first $\vartheta$ sum is $\geq 0$, with equality iff the sequence $\left|x_{i}\right|$ is constant; by the case $d_{i} \equiv 0$ of Lemma 3.3. the second $\vartheta$ sum is $\geq 0$, with equality iff at most one of the $x_{i}$ is nonzero. It follows that the integrand is exponentially small unless both conditions are satisfied; i.e., unless $x_{i} \equiv 0$. The remainder of the proof is as above. 
Remark. Note that it also follows from the above proof that the convergence condition is necessary for the integrand to be localized. One can readily arrange for equality to hold in the first sum, but not the second, at which point if $\Im\left(-\left((n-1) \tau+\omega_{1}+\omega_{2}\right) / \omega_{1} \omega\right)<0$, the integrand is exponentially larger than its value near $x_{i} \equiv 0$.

Let $I I_{B C_{n} ; h}^{(m)}\left(\mu_{0}, \ldots ; \tau ; \omega_{1}, \omega_{2}\right)$ denote the above hyperbolic integral, as a meromorphic function on the domain

$$
\Im(\tau), \Im\left(\omega_{1}\right), \Im\left(\omega_{2}\right), \Im\left(\frac{-(n-1) \tau-\omega_{1}-\omega_{2}}{\omega_{1} \omega_{2}}\right)>0, \quad(2 n-2) \tau+\sum_{r} \mu_{r}=(m+1)\left(\omega_{1}+\omega_{2}\right) .
$$

Corollary 4.4. Let $\mu_{0}, \mu_{1}, \ldots, \mu_{5}, \tau, \omega_{1}, \omega_{2}$ be parameters such that

$$
\Im(\tau), \Im\left(\omega_{1}\right), \Im\left(\omega_{2}\right)>0, \quad(2 n-2) \tau+\sum_{r} \mu_{r}=\omega_{1}+\omega_{2},
$$

and satisfying the convergence condition

$$
\Im\left(\frac{-(n-1) \tau-\omega_{1}-\omega_{2}}{\omega_{1} \omega_{2}}\right)>0 .
$$

Then

$$
I I_{B C_{n} ; h}^{(0)}\left(\mu_{0}, \ldots, \mu_{5} ; \tau ; \omega_{1}, \omega_{2}\right)=\prod_{0 \leq i<n} \Gamma_{h}\left((i+1) \tau ; \omega_{1}, \omega_{2}\right) \prod_{0 \leq r<s \leq 5} \Gamma_{h}\left(i \tau+\mu_{r}+\mu_{s} ; \omega_{1}, \omega_{2}\right) .
$$

Corollary 4.5. For parameters $\mu_{0}, \ldots, \mu_{7}, \tau, \omega_{1}, \omega_{2}$ such that there exists an integer $n$ (necessarily unique) with

$$
(2 n+2) \tau+\sum_{r} \mu_{r}=2\left(\omega_{1}+\omega_{2}\right)
$$

and

$$
\Im(\tau), \Im\left(\omega_{1}\right), \Im\left(\omega_{2}\right), \Im\left(\frac{-(n-1) \tau-\omega_{1}-\omega_{2}}{\omega_{1} \omega_{2}}\right)>0
$$

define

$$
\tilde{I I}_{h}\left(\mu_{0}, \ldots, \mu_{7} ; \tau ; \omega_{1}, \omega_{2}\right):=\left(\prod_{0 \leq r<s \leq 7} \Gamma_{h}^{+}\left(\tau+\mu_{r}+\mu_{s} ; \tau, \omega_{1}, \omega_{2}\right)\right) I I_{h}^{(1)}\left(\tau / 2+\mu_{0}, \ldots, \tau / 2+\mu_{7} ; \tau ; \omega_{1}, \omega_{2}\right) .
$$

Then $\tilde{I}_{h}$ is invariant under the natural action of the Weyl group $E_{7}$; in other words, it satisfies the identities

$$
\tilde{I I}_{h}\left(\mu_{0}, \ldots, \mu_{7} ; \tau ; \omega_{1}, \omega_{2}\right)=\tilde{I}_{h}\left(\mu_{0}+\nu, \ldots, \mu_{3}+\nu, \mu_{4}-\nu, \ldots, \mu_{7}-\nu ; \tau ; \omega_{1}, \omega_{2}\right)
$$

where $\nu=\left(\mu_{4}+\mu_{5}+\mu_{6}+\mu_{7}-\mu_{0}-\mu_{1}-\mu_{2}-\mu_{3}\right) / 4$;

$$
\tilde{I}_{h}\left(\mu_{0}, \ldots, \mu_{7} ; \tau ; \omega_{1}, \omega_{2}\right)=\tilde{I}_{h}\left(\nu-\mu_{0}, \ldots, \nu-\mu_{3}, \nu^{\prime}-\mu_{4}, \ldots, \nu^{\prime}-\mu_{7} ; \tau ; \omega_{1}, \omega_{2}\right),
$$

where $\nu=\left(\mu_{0}+\mu_{1}+\mu_{2}+\mu_{3}\right) / 2, \nu^{\prime}=\left(\mu_{4}+\mu_{5}+\mu_{6}+\mu_{7}\right) / 2 ;$ and

$$
\tilde{I I}_{h}\left(\mu_{0}, \ldots, \mu_{7} ; \tau ; \omega_{1}, \omega_{2}\right)=\tilde{I I}_{h}\left(\nu-\mu_{0}, \ldots, \nu-\mu_{7} ; \tau ; \omega_{1}, \omega_{2}\right),
$$

where $\nu=\left(\mu_{0}+\mu_{1}+\cdots+\mu_{7}\right) / 2$; as well as invariance under permutations of $\mu_{0}$ through $\mu_{7}$. 
Remark. Similarly, the other double coset of $E_{7}$ in $E_{8}$ that gives rise to (dimension-altering) transformations of the elliptic integral also gives rise to transformations of the hyperbolic integral; we omit the obvious details. The key observation is that the overall exponential factor that arises when taking the limit is, once one solves for $n$, a function of $\sum_{i} \mu_{i}^{2}$, and is thus $E_{8}$-invariant. The work of [15] on recurrences also descends to the hyperbolic case; in particular, for $\tau=\omega_{2}$, one obtains a tau-function for a hyperbolic analogue of the elliptic Painlevé equation.

For the $A_{n}$ integral, the difficulty is that the elliptic integral has a condition $\prod_{i} z_{i}=1$, which in $x_{i}$ coordinates, becomes $\sum_{i} x_{i} \in \mathbb{Z}$; this introduces extra complications when maximizing the integrand. Recall that the $A_{n}$ integral is defined by

$$
\begin{aligned}
& I_{A_{n}}^{(m)}\left(u_{0}, \ldots u_{m+n+1} ; v_{0}, \ldots v_{m+n+1} ; p, q\right) \\
& \quad:=\frac{(p ; p)^{n}(q ; q)^{n}}{(n+1) !} \int_{\prod_{0 \leq i \leq n} z_{i}=1} \frac{\prod_{0 \leq i \leq n} \prod_{0 \leq r<m+n+2} \Gamma_{e}\left(u_{r} z_{i}, v_{r} / z_{i} ; p, q\right)}{\prod_{0 \leq i<j \leq n} \Gamma_{e}\left(z_{i} / z_{j}, z_{j} / z_{i} ; p, q\right)} \prod_{1 \leq i \leq n} \frac{d z_{i}}{2 \pi \sqrt{-1} z_{i}},
\end{aligned}
$$

for $0<|p|,|q|<1,0<\left|u_{0}\right|, \ldots,\left|u_{m+n+1}\right|,\left|v_{0}\right|, \ldots,\left|v_{m+n+1}\right|<1, \prod_{i} u_{i} v_{i}=(p q)^{m+1}$. (It follows from general principles that this can be extended to a meromorphic function on the domain $0<|p|,|q|<1, \prod_{i} u_{i} v_{i}=$ $(p q)^{m+1}$, but the condition on the contour is complicated.)

Theorem 4.6. Let $\mu_{0}, \mu_{1}, \ldots, \mu_{m+n+1}, \nu_{0}, \nu_{1}, \ldots, \nu_{m+n+1}, \omega_{1}, \omega_{2}$ be parameters in the upper half-plane such that

$$
\sum_{r} \mu_{r}+\nu_{r}=(m+1)\left(\omega_{1}+\omega_{2}\right)
$$

Then as $v \rightarrow 0^{+}$,

$$
\begin{array}{r}
e\left(-(n+1) \sum_{r}\left(R\left(v \mu_{r} ; v \omega_{1}, v \omega_{2}\right)+R\left(v \nu_{r} ; v \omega_{1}, v \omega_{2}\right)\right)+\left(n^{2}+2 n\right) R\left(0 ; v \omega_{1}, v \omega_{2}\right)\right) \\
\cdot I_{A_{n}}^{(m)}\left(e\left(v \mu_{0}\right), e\left(v \mu_{1}\right), \ldots ; e\left(v \nu_{0}\right), e\left(v \nu_{1}\right), \ldots ; e\left(v \omega_{1}\right), e\left(v \omega_{2}\right)\right)
\end{array}
$$

converges uniformly exponentially (over compact subsets) to

$$
\frac{1}{\left(\sqrt{-\omega_{1} \omega_{2}}\right)^{n}(n+1) !} \int_{\sum_{0 \leq i \leq n} x_{i}=0} \frac{\prod_{0 \leq i \leq n} \prod_{0 \leq r<m+n+2} \Gamma_{h}\left(\mu_{r}+x_{i}, \nu_{r}-x_{i} ; \omega_{1}, \omega_{2}\right)}{\prod_{0 \leq i<j \leq n} \Gamma_{h}\left(x_{i}-x_{j}, x_{j}-x_{i} ; \omega_{1}, \omega_{2}\right)} \prod_{1 \leq i \leq n} d x_{i} .
$$

Proof. If we perform the change of variables $z_{i}=e\left(x_{i}\right)$ in the elliptic integral, the result is an integral over the domain

$$
-1 / 2 \leq x_{0}, x_{1}, x_{2}, \ldots, x_{n} \leq 1 / 2 ; \quad \sum_{0 \leq i \leq n} x_{i} \in Z,
$$

a disjoint union of polytopes. Over the entire cube, we find that the integrand is uniformly

$$
O\left(e\left(\frac{-\omega_{1}-\omega_{2}}{2 v \omega_{1} \omega_{2}}\left(\sum_{0 \leq i \leq n}(n+1) \vartheta\left(x_{i}\right)-\sum_{0 \leq i<j \leq n} \vartheta\left(x_{i}-x_{j}\right)\right)\right)\right) .
$$

Now, we find from the case $d_{i} \equiv 0$ of Lemma 3.2 that

$$
\sum_{0 \leq i \leq n}(n+1) \vartheta\left(x_{i}\right)-\sum_{0 \leq i<j \leq n} \vartheta\left(x_{i}-x_{j}\right) \geq \vartheta\left(\sum_{i} x_{i}\right) \geq 0
$$


with equality iff $x_{0}, \ldots, x_{n}$ interlaces with $0, \ldots, 0$ and $\sum_{i} x_{i} \in \mathbb{Z}$; i.e., iff $x_{i} \equiv 0$. We thus conclude that the integral over the polytope

$$
-1 / 4 \leq x_{0}, x_{1}, \ldots, x_{n} \leq 1 / 4 ; \quad \sum_{i} x_{i}=0
$$

is uniformly exponentially close to the original integral. Thus, as above, the theorem reduces to showing that the hyperbolic integral decays exponentially. This in turn reduces to the identity

$$
(n+1) \sum_{0 \leq i \leq n}\left|x_{i}\right|-\sum_{0 \leq i<j \leq n}\left|x_{i}-x_{j}\right| \geq 0
$$

with equality only when $x_{1}=x_{2}=\cdots x_{n}=0$.

The remaining issue in degenerating [16] to the hyperbolic level is the degeneration of the biorthogonal functions constructed there. The primary difficulty is that the construction of those functions in [16] does not give rise to good uniform asymptotics. However, we can still establish the following.

Theorem 4.7. Let the parameters $\tau_{0}, \tau_{1}, \tau_{2}, \tau_{3}, \mu_{0}, \mu_{1}, \tau, \omega_{1}, \omega_{2}$ be parameters with $\tau, \omega_{1}, \omega_{2}$ in the upper half-plane such that

$$
(2 n-2) \tau+\tau_{0}+\tau_{1}+\tau_{2}+\tau_{3}+\mu_{0}+\mu_{1}=\omega_{1}+\omega_{2} .
$$

Then for any partition pair $\boldsymbol{\lambda}$, and for generic values of the parameters, the biorthogonal function

$$
\tilde{\mathcal{R}}_{\boldsymbol{\lambda}}^{(n)}\left(\ldots, e\left(x_{i}\right), \ldots ; e\left(v \tau_{0}\right): e\left(v \tau_{1}\right), e\left(v \tau_{2}\right), e\left(v \tau_{3}\right) ; e\left(v \mu_{0}\right), e\left(v \mu_{1}\right) ; e(v \tau) ; e\left(v \omega_{1}\right), e\left(v \omega_{2}\right)\right)
$$

is uniformly bounded for $\left(x_{1}, \ldots, x_{n}\right) \in D(v)^{n}$, where $D(v)$ is a region of the form $-1-v C_{1} \leq \Re(x) \leq v C_{1}$, $|\Im(x)| \leq v C_{2}$, and excluding a hole of radius $\epsilon v$ around every pole of the biorthogonal function. Moreover, there exists a function

$$
\tilde{\mathcal{R}}_{\boldsymbol{\lambda} ; h}^{(n)}\left(\ldots, x_{i}, \ldots ; \tau_{0}: \tau_{1}, \tau_{2}, \tau_{3} ; \mu_{0}, \mu_{1} ; \tau ; \omega_{1}, \omega_{2}\right)
$$

such that as $v \rightarrow 0^{+}$,

$$
\begin{aligned}
& \tilde{\mathcal{R}}_{\boldsymbol{\lambda}}^{(n)}\left(\ldots, e\left(x_{i}\right), \ldots ; e\left(v \tau_{0}\right): e\left(v \tau_{1}\right), e\left(v \tau_{2}\right), e\left(v \tau_{3}\right) ; e\left(v \mu_{0}\right), e\left(v \mu_{1}\right) ; e(v \tau) ; e\left(v \omega_{1}\right), e\left(v \omega_{2}\right)\right) \\
- & \tilde{\mathcal{R}}_{\boldsymbol{\lambda} ; h}^{(n)}\left(\ldots, x_{i} / v, \ldots ; \tau_{0}: \tau_{1}, \tau_{2}, \tau_{3} ; \mu_{0}, \mu_{1} ; \tau ; \omega_{1}, \omega_{2}\right)
\end{aligned}
$$

converges exponentially to 0, uniformly for $x$ in a compact subset of the domain $-1<\Re(x)<1$.

Proof. We first observe that the claims of the theorem are certainly true if we replace $\tilde{\mathcal{R}}_{\lambda}^{(n)}$ by a product of functions of the form

$$
e\left(2 n\left(R\left(v \beta ; v \omega_{1}\right)-R\left(v \alpha ; v \omega_{1}\right)\right)\right) \prod_{1 \leq i \leq n} \frac{\theta\left(e(v \alpha) z_{i}^{ \pm 1} ; e\left(v \omega_{1}\right)\right)}{\theta\left(e(v \beta) z_{i}^{ \pm 1} ; e\left(v \omega_{1}\right)\right)},
$$

or similarly for $\omega_{2}$. In particular, it was established in [16] that there exist functions $F_{\boldsymbol{\lambda}}^{(n)}\left(\mu_{0}: ; \tau ; \omega_{1}, \omega_{2}\right)$ of the above form such that there exists an expansion

$$
\tilde{\mathcal{R}}_{\lambda}^{(n)}=\sum_{\mu \subset \boldsymbol{\lambda}} C_{\boldsymbol{\lambda} \mu} F_{\boldsymbol{\lambda}}
$$


for some coefficients $C_{\boldsymbol{\lambda} \mu}$ independent of $z_{i}$. It thus remains only to show that for generic parameters, these coefficients $C_{\boldsymbol{\lambda} \mu}$ converge exponentially. Moreover, the action of the integral operators of [16] can be computed explicitly in the $F_{\boldsymbol{\lambda}}$ basis, and the coefficients of the corresponding matrices converge exponentially (to a triangular matrix with generically nonzero diagonal). Thus the generalized eigenvalue equations satisfied by $\tilde{\mathcal{R}}_{\boldsymbol{\lambda}}^{(n)}$ set up linear equations in the $C_{\boldsymbol{\lambda} \boldsymbol{\mu}}$ with exponentially converging coefficients. Since the limits of the generalized eigenvalues are generically distinct, the limiting linear equations are generically nonsingular, and the result follows.

Remark. The unviariate hyperbolic biorthogonal function

$$
\tilde{\mathcal{R}}_{\boldsymbol{\lambda} ; h}^{(1)}\left(x ; \tau_{0}: \tau_{1}, \tau_{2}, \tau_{3} ; \mu_{0}, \mu_{1} ; \tau ; \omega_{1}, \omega_{2}\right)
$$

was discussed in [20, §8.3].

Note in particular that if we multiply the integrand of either $B C_{n}$ integral by a function satisfying such convergence properties, the resulting integral will also converge exponentially (assuming the unadorned integral so converges). Also, a similar argument works for the interpolation functions (which as special cases of the biorthogonal functions do not quite fall under the above generic result, but again satisfy suitable integral equations). As a result, every identity of [16] involving such functions converges exponentially (possibly with an explicit factor of the form $\exp (a+b v))$ to a corresponding hyperbolic limit. One should note (as observed in 20 , $\S 8.3])$ that further degeneration of the parameters can lead to convergence issues, as without the moderating effect of the poles, the biorthogonal functions grow exponentially as $|\Re(x)| \rightarrow \infty$.

\section{$5 \quad$ Trigonometric limits}

The main difficulty with the trigonometric limit $p \rightarrow 0$ is that the general case of the transformations involves parameters tending to infinity, making the contour ill-behaved in the limit. This can be fixed at the expense of breaking the symmetry of the integrand.

Recall that for the type $I B C_{n}$ integral, the parameters are constrained to satisfy the balancing condition

$$
\prod_{0 \leq r \leq 2 n+2 m+3} u_{r}=(p q)^{m+1} .
$$

The natural way to satisfy this in the $p \rightarrow 0$ limit is for $2 n+m+3$ of the parameters to be $\Theta(1)$, while the remaining $m+1$ parameters are $\Theta(p)$. This then makes the $p \rightarrow 0$ limit of the integral trivial to compute.

Theorem 5.1. For any parameters $u_{0}, \ldots, u_{2 n+m+2}, v_{0}, \ldots, v_{m}, q$ satisfying

$$
|q|<1, \quad \prod_{0 \leq r \leq 2 n+m+2} u_{r}=\prod_{0 \leq r \leq m} v_{r},
$$

we have the limit

$$
\begin{aligned}
\lim _{p \rightarrow 0} I_{B C_{n}}^{(m)}\left(u_{0}, \ldots, u_{2 n+m+2}, p q / v_{0}, \ldots, p q / v_{m} ; p, q\right) & \\
= & \frac{(q ; q)^{n}}{2^{n} n !} \int_{C^{n}} \prod_{1 \leq i<j \leq n} \Gamma_{t}\left(z_{i}^{ \pm 1} z_{j}^{ \pm 1} ; q\right)^{-1} \prod_{1 \leq i \leq n} \frac{\prod_{0 \leq r \leq 2 n+m+2} \Gamma_{t}\left(u_{r} z_{i}^{ \pm 1} ; q\right)}{\Gamma_{t}\left(z_{i}^{ \pm 2} ; q\right) \prod_{0 \leq r \leq m} \Gamma_{t}\left(v_{r} z_{i}^{ \pm 1} ; q\right)} \frac{d z_{i}}{2 \pi \sqrt{-1} z_{i}},
\end{aligned}
$$


where the contour contains all points of the form $p^{i} q^{j} u_{r}, i, j \geq 0$, and excludes their reciprocals.

Proof. This follows immediately from the facts that as $p \rightarrow 0$,

$$
\begin{aligned}
\Gamma_{e}(x ; p, q)^{ \pm 1} & =\Gamma_{t}(x ; q)^{ \pm 1}(1+O(p)) \\
\Gamma_{e}(p q / x ; p, q)^{ \pm 1} & =\Gamma_{t}(x ; q)^{\mp 1}(1+O(p)),
\end{aligned}
$$

uniformly in $x$ away from the poles.

Unfortunately, the right-hand side of the type I transformation involves parameters

$$
(p q)^{1 / 2} / u_{0}, \ldots,(p q)^{1 / 2} / u_{2 n+m+2},(p q)^{-1 / 2} v_{0}, \ldots,(p q)^{-1 / 2} v_{m}
$$

which as mentioned above gives an apparently ill-behaved limit. The primary difficulty is that the divergent parameters not only deform the contour, but in fact pinch the contour in the limit, making it approach both 0 and infinity. It turns out, however, that there is a way to break the symmetry in such a way as to eliminate half of the offending poles, thus allowing the contour to be renormalized, giving a well-behaved limit.

The key fact is the following identity of $q$-elliptic functions. Here $R\left(z_{i}\right)$ denotes the operator such that $R\left(z_{i}\right) f\left(z_{i}\right)=f\left(1 / z_{i}\right)$.

Lemma 5.2. For any parameters $u_{0}, \ldots, u_{n+1}, q$ we have the identity

$$
\prod_{1 \leq i \leq n}\left(1+R\left(z_{i}\right)\right) \frac{\theta\left(\prod_{0 \leq r \leq n+1} u_{r} / \prod_{1 \leq i \leq n} z_{i} ; q\right) \prod_{1 \leq i \leq n} \prod_{0 \leq r \leq n+1} \theta\left(u_{r} z_{i} ; q\right)}{\prod_{1 \leq i \leq j \leq n} \theta\left(z_{i} z_{j} ; q\right)}=\prod_{0 \leq r<s \leq n+1} \theta\left(u_{r} u_{s} ; q\right) .
$$

Proof. The left-hand side can be expressed as a sum of $2^{n}$ terms, all of which are elliptic functions in $z$ with respect to multiplication by $q$, and thus the sum is also an elliptic function. Moreover, since the original function is invariant under permutations, the sum is invariant under the action of $B C_{n}$. In particular, the order of the sum along each reflection hyperplane must be even; since the summands have at most simple poles there, it follows that the sum is constant. The constant can be recovered by taking $z_{i}=u_{i}$, making all but one summand vanish.

Lemma 5.3. For any nonzero parameters $t_{0}, \ldots, t_{n}, u_{0}, \ldots, u_{n+m+1}, v_{0}, \ldots, v_{m}, p, q$ with

$$
0<|p|,|q|<1, \quad \prod_{0 \leq r \leq n} t_{r}=\prod_{0 \leq r \leq n+m+1} u_{r} \prod_{0 \leq r \leq m} v_{r}
$$

and any complex parameters $a, w \neq 0$, we have the identity

$$
\begin{gathered}
\prod_{0 \leq r<s \leq n} \theta\left(t_{r} t_{s} / a ; q\right) I_{B C_{n}}^{(m)}\left(t_{0} / a^{1 / 2}, \ldots, t_{n} / a^{1 / 2}, a^{1 / 2} / u_{0}, \ldots, a^{1 / 2} / u_{m+n+1}, p q / a^{1 / 2} v_{0}, \ldots, p q / a^{1 / 2} v_{m} ; p, q\right) \\
=\frac{(p ; p)^{n}(q ; q)^{n}}{n !} \int_{C^{n}} \frac{\theta\left(\prod_{0 \leq r \leq n} t_{r} / w \prod_{1 \leq i \leq n} z_{i} ; q\right) \prod_{1 \leq i \leq n} \theta\left(z_{i} / w ; q\right)}{\prod_{0 \leq r \leq n} \theta\left(t_{r} / w ; q\right)} \frac{\prod_{1 \leq i \leq j \leq n} \theta\left(p z_{i} z_{j} / a ; p\right)}{\prod_{1 \leq i<j \leq n} \Gamma_{e}\left(\left(z_{i} / z_{j}\right)^{ \pm 1} ; p, q\right)} \\
\prod_{1 \leq i \leq n} \prod_{0 \leq r \leq n} \Gamma_{e}\left(p t_{r} z_{i} / a, t_{r} / z_{i} ; p, q\right) \prod_{0 \leq r \leq m+n+1} \frac{\Gamma_{e}\left(z_{i} / u_{r} ; p, q\right)}{\Gamma_{e}\left(p q z_{i} u_{r} / a ; p, q\right)} \\
\prod_{0 \leq r \leq m} \frac{\Gamma_{e}\left(p q z_{i} / a v_{r} ; p, q\right)}{\Gamma_{e}\left(z_{i} v_{r} ; p, q\right)} \frac{d z_{i}}{2 \pi \sqrt{-1} z_{i}}
\end{gathered}
$$


where the contour contains all points of the form $p^{i} q^{j} t_{r}, p^{i} q^{j} a / u_{r}, p^{i+1} q^{j+1} / v_{r}, i, j \geq 0$, and excludes all points of the form $p^{-1-i} q^{-j} a / t_{r}, p^{-i} q^{-j} u_{r}, p^{-i-1} q^{-j-1} a v_{r}, i, j \geq 0$.

Proof. If we multiply the integrand on the left-hand side by the case $u_{r}=a^{-1 / 2} v_{r}, 0 \leq r \leq n, u_{n+1}=a^{1 / 2} / w$ of the lemma, the symmetry of the integrand implies that $\prod_{1 \leq i \leq n}\left(1+R\left(z_{i}\right)\right)$ can be replaced by $2^{n}$. Shifting the variables of integration by $z_{i} \rightarrow a^{-1 / 2} z_{i}$ gives the right-hand side, up to a shift in contour with no effect on the integral.

Remark 1. Note that for specific choices of $w$, the contour condition may conceivably be weakened; the point is that the $w$-dependent factors can cancel out poles of the integrand, making the corresponding constraints on the contour superfluous. In particular, for certain specializations of the parameters, it can be the case that the contour conditions for generic $w$ are inconsistent, but a suitable choice of $w$ makes the integral well-defined.

Remark 2. If we multiply the integrand on the left by a symmetric function $f$ (adjusting the contour conditions accordingly), the effect is to multiply the nonsymmetric integrand by $f\left(\ldots a^{-1 / 2} z_{i} \ldots\right)$, with suitable contour conditions.

This makes the limit $p \rightarrow 0$ trivial again, as long as $|p q| \leq|a|<1$. Taking $a=p q$ gives the following.

Theorem 5.4. For any nonzero parameters $u_{0}, \ldots, u_{2 n+m+2}, v_{0}, \ldots, v_{m}, q$ with

$$
0<|q|<1, \quad \prod_{0 \leq r \leq 2 n+m+2} u_{r}=\prod_{0 \leq r \leq m} v_{r}
$$

we have the limit

$$
\begin{gathered}
\lim _{p \rightarrow 0} \prod_{0 \leq r<s \leq m} \theta\left(v_{r} v_{s} / p q ; q\right) I_{B C_{m}}^{(n)}\left((p q)^{1 / 2} / u_{0}, \ldots,(p q)^{1 / 2} / u_{2 n+m+2},(p q)^{-1 / 2} v_{0}, \ldots,(p q)^{-1 / 2} v_{m} ; p, q\right) \\
=\frac{(q ; q)^{m}}{m !} \int_{C^{m}} \frac{\theta\left(\prod_{0 \leq r \leq m} v_{r} / w \prod_{1 \leq i \leq m} z_{i} ; q\right) \prod_{1 \leq i \leq m} \theta\left(z_{i} / w ; q\right)}{\prod_{0 \leq r \leq m} \theta\left(v_{r} / w ; q\right)} \frac{\prod_{1 \leq i \leq j \leq m}\left(1-z_{i} z_{j} / q\right)}{\prod_{1 \leq i<j \leq m} \Gamma_{t}\left(\left(z_{i} / z_{j}\right)^{ \pm 1} ; q\right)} \\
\prod_{1 \leq i \leq m} \prod_{0 \leq r \leq 2 n+m+2} \frac{\Gamma_{t}\left(z_{i} / u_{r} ; q\right)}{\Gamma_{t}\left(z_{i} u_{r} ; q\right)} \prod_{0 \leq r \leq m} \Gamma_{t}\left(v_{r} z_{i} / q, v_{r} / z_{i} ; q\right) \frac{d z_{i}}{2 \pi \sqrt{-1} z_{i}},
\end{gathered}
$$

where the contour contains all points of the form $q^{j} v_{r}, j \geq 0$, and excludes all points of the form $q^{-j} u_{r}, q^{1-j} / v_{r}$, $j \geq 0$.

Corollary 5.5. The trigonometric integral of Theorem 5.1 is equal to

$$
\prod_{0 \leq r<s \leq 2 n+m+2} \Gamma_{t}\left(u_{r} u_{s} ; q\right) \prod_{\substack{0 \leq r \leq 2 n+m+2 \\ 0 \leq s \leq m}} \Gamma_{t}\left(v_{s} / u_{r} ; q\right)^{-1} \prod_{0 \leq r<s \leq m} \Gamma_{t}\left(v_{r} v_{s} / q ; q\right)^{-1}
$$

times the trigonometric integral of Theorem 5.4 .

Remark. The univariate cases $n=1, m=0$ and $n=0, m=1$ are the Nasrallah-Rahman integral and an integral identity of Gasper (equations (6.4.1) and (4.11.4) of [8]); the general $m=0$ case is due to Gustafson [9]. 
We also obtain a nontrivial transformation by taking $a \sim p^{\alpha}$ for $0<\alpha<1$, say $a=(p q)^{1 / 2}$ for symmetry.

Theorem 5.6. For any nonzero parameters $t_{0}, \ldots, t_{n}, u_{0}, \ldots, u_{n+m+1}, v_{0}, \ldots, v_{m}, q$ with

$$
0<|q|<1, \quad \prod_{0 \leq r \leq n} t_{r}=\prod_{0 \leq r \leq n+m+1} u_{r} \prod_{0 \leq r \leq m} v_{r}
$$

and any complex parameter $w$, we have the limit

$$
\begin{gathered}
\lim _{p \rightarrow 0} \prod_{0 \leq r<s \leq n} \theta\left((p q)^{-1 / 2} t_{r} t_{s} ; q\right) I_{B C_{n}}^{(m)}\left(\frac{t_{0}}{(p q)^{1 / 4}}, \ldots, \frac{t_{n}}{(p q)^{1 / 4}}, \frac{(p q)^{1 / 4}}{u_{0}}, \ldots, \frac{(p q)^{1 / 4}}{u_{m+n+1}}, \frac{(p q)^{3 / 4}}{v_{0}}, \ldots, \frac{(p q)^{3 / 4}}{v_{m}} ; p, q\right) \\
=\frac{(q ; q)^{n}}{n !} \int_{C^{n}} \frac{\theta\left(\prod_{0 \leq r \leq n} t_{r} / w \prod_{1 \leq i \leq n} z_{i} ; q\right) \prod_{1 \leq i \leq n} \theta\left(z_{i} / w ; q\right)}{\prod_{0 \leq r \leq n} \theta\left(t_{r} / w ; q\right)} \prod_{1 \leq i<j \leq n} \Gamma_{t}\left(\left(z_{i} / z_{j}\right)^{ \pm 1} ; q\right)^{-1} \\
\prod_{1 \leq i \leq n} \frac{\prod_{0 \leq r \leq n} \Gamma_{t}\left(t_{r} / z_{i} ; q\right) \prod_{0 \leq r \leq m+n+1} \Gamma_{t}\left(z_{i} / u_{r} ; q\right)}{\prod_{0 \leq r \leq m} \Gamma_{t}\left(z_{i} v_{r} ; q\right)} \frac{d z_{i}}{2 \pi \sqrt{-1} z_{i}}
\end{gathered}
$$

where the contour contains all points of the form $q^{i} t_{r}, i \geq 0$, and excludes all points of the form $q^{-i} u_{r}, i \geq 0$.

Corollary 5.7. The trigonometric integral of Theorem 5.6 is independent of $w$, and if multiplied by

$$
\prod_{0 \leq r \leq n} \prod_{0 \leq s \leq n+m+1} \Gamma_{t}\left(t_{r} / u_{s} ; q\right)^{-1}
$$

is invariant under the involution

$$
\left(m, n ; \ldots, t_{r}, \ldots ; \ldots, u_{r}, \ldots ; \ldots, v_{r} \ldots\right) \rightarrow\left(n, m ; \ldots, v_{r} \ldots ; \ldots, u_{r}^{-1}, \ldots ; \ldots, t_{r}, \ldots\right)
$$

Remark. This can also be obtained as a limit of Corollary 5.5 after first breaking the symmetry of the lefthand side as in Lemma 5.3. In particular, this should perhaps be thought of as a degeneration rather than a direct limit; we mention it to point out that that distinction is somewhat artificial (any degeneration should be obtainable as a limit directly from the elliptic level), but more importantly because the Type II analogue has important consequences.

For the Type II integral, we again have a trivial limit in one case.

Theorem 5.8. For any parameters $u_{0}, \ldots, u_{m+4}, v_{0}, \ldots, v_{m}, q$ satisfying

$$
|q|<1, \quad t^{2 n-2} \prod_{0 \leq r \leq m+4} u_{r}=\prod_{0 \leq r \leq m} v_{r}
$$

we have the limit

$$
\begin{aligned}
\lim _{p \rightarrow 0} I I_{B C_{n}}^{(m)} & \left(u_{0}, \ldots, u_{m+4}, p q / v_{0}, \ldots, p q / v_{m} ; t ; p, q\right) \\
= & \frac{(q ; q)^{n} \Gamma_{t}(t ; q)^{n}}{2^{n} n !} \int_{C^{n}} \prod_{1 \leq i<j \leq n} \frac{\Gamma_{t}\left(t z_{i}^{ \pm 1} z_{j}^{ \pm 1} ; q\right)}{\Gamma_{t}\left(z_{i}^{ \pm 1} z_{j}^{ \pm 1} ; q\right)} \prod_{1 \leq i \leq n} \frac{\prod_{0 \leq r \leq m+4} \Gamma_{t}\left(u_{r} z_{i}^{ \pm 1} ; q\right)}{\Gamma_{t}\left(z_{i}^{ \pm 2} ; q\right) \prod_{0 \leq r \leq m} \Gamma_{t}\left(v_{r} z_{i}^{ \pm 1} ; q\right)} \frac{d z_{i}}{2 \pi \sqrt{-1} z_{i}},
\end{aligned}
$$

where the contour $C$ satisfies $C=C^{-1}$, and for all $i \geq 0$, contains the points $q^{i} u_{r}$ as well as the contour $q^{i} t C$. 
We recall from [16] the following identity, which plays the role of Lemma 5.2 for the type II $B C_{n}$ integral.

$$
\prod_{1 \leq i \leq n}\left(1+R\left(z_{i}\right)\right) \frac{\theta\left(u_{0} z_{i}, u_{1} z_{i}, u_{2} z_{i}, t^{n-1} u_{0} u_{1} u_{2} / z_{i} ; q\right)}{\theta\left(z_{i}^{2} ; q\right)} \prod_{1 \leq i<j \leq n} \frac{\theta\left(t z_{i} z_{j} ; q\right)}{\theta\left(z_{i} z_{j} ; q\right)}=\prod_{0 \leq i<n} \theta\left(t^{i} u_{0} u_{1}, t^{i} u_{0} u_{2}, t^{i} u_{1} u_{2} ; q\right) .
$$

Lemma 5.9. For any nonzero parameters $t_{0}, t_{1}, u_{0}, \ldots, u_{m+1}, v_{0}, \ldots, v_{m}, p, q$ with

$$
0<|p|,|q|<1, \quad t^{2 n-2} t_{0} t_{1}=\prod_{0 \leq r \leq m+2} u_{r} \prod_{0 \leq r \leq m} v_{r}
$$

and any complex parameters $a, w \neq 0$, we have the identity

$$
\begin{gathered}
\prod_{0 \leq i<n} \theta\left(t^{i} t_{0} t_{1} / a ; q\right) I I_{B C_{n}}^{(m)}\left(t_{0} / a^{1 / 2}, t_{1} / a^{1 / 2}, a^{1 / 2} / u_{0}, \ldots, a^{1 / 2} / u_{m+2}, p q / a^{1 / 2} v_{0}, \ldots, p q / a^{1 / 2} v_{m} ; t ; p, q\right) \\
=\frac{(p ; p)^{n}(q ; q)^{n} \Gamma_{e}(t ; p, q)^{n}}{n !} \int_{C^{n}} \prod_{1 \leq i \leq n} \frac{\theta\left(z_{i} / w, t^{n-1} t_{0} t_{1} / w z_{i} ; q\right)}{\theta\left(t^{i-1} t_{0} / w, t^{i-1} t_{1} / w ; q\right)} \prod_{1 \leq i<j \leq n} \frac{\Gamma_{e}\left(t p z_{i} z_{j} / a, p q z_{i} z_{j} / a, t\left(z_{i} / z_{j}\right)^{ \pm 1} ; p, q\right)}{\Gamma_{e}\left(p z_{i} z_{j} / a, p q z_{i} z_{j} / t a,\left(z_{i} / z_{j}\right)^{ \pm 1} ; p, q\right)} \\
\prod_{1 \leq i \leq n} \theta\left(p z_{i}^{2} / a ; p\right) \Gamma_{e}\left(p t_{0} z_{i} / a, p t_{1} z_{i} / a, t_{0} / z_{i}, t_{1} / z_{i} ; p, q\right) \\
\prod_{0 \leq r \leq m+2} \frac{\Gamma_{e}\left(z_{i} / u_{r} ; p, q\right)}{\Gamma_{e}\left(p q z_{i} u_{r} / a ; p, q\right)} \prod_{0 \leq r \leq m} \frac{\Gamma_{e}\left(p q z_{i} / a v_{r} ; p, q\right)}{\Gamma_{e}\left(z_{i} v_{r} ; p, q\right)} \frac{d z_{i}}{2 \pi \sqrt{-1} z_{i}},
\end{gathered}
$$

where the contour $C$ is chosen so that for all $i, j \geq 0$, it contains the points and contours

$$
p^{i} q^{j} t_{0}, p^{i} q^{j} t_{1}, p^{i} q^{j} a / u_{r}, p^{i+1} q^{j+1} / v_{r}, \quad p^{i} q^{j} t C, p^{i} q^{j} t a / C
$$

and excludes the points and contours

$$
a / p^{i+1} q^{j} t_{0}, a / p^{i+1} q^{j} t_{1}, u_{r} / p^{i} q^{j}, a v_{r} / p^{i+1} q^{j+1}, \quad a / p^{i+1} q^{j} t C, C / p^{i} q^{j} t .
$$

Remark. It is possible to choose a contour of the given form satisfying $C=a C^{-1}$, namely $a^{-1} C_{0}$ where $C_{0}$ is a suitable contour for the left-hand side.

Theorem 5.10. For any nonzero parameters $u_{0}, \ldots, u_{2 m+3}, v_{0}, v_{1}, q$ with

$$
0<|q|<1, \quad \prod_{0 \leq r \leq 2 m+3} u_{r}=t^{2 n-2} v_{0} v_{1}
$$

and any complex parameters $w \neq 0$, we have the identity

$$
\begin{aligned}
\lim _{p \rightarrow 0} \prod_{0 \leq i<n} \theta\left(t^{i} v_{0} v_{1} / p q ; q\right) I I_{B C_{n}}^{(m)}\left(v_{0} /(p q)^{1 / 2}, v_{1} /(p q)^{1 / 2},(p q)^{1 / 2} / u_{0}, \ldots,(p q)^{1 / 2} / u_{2 m+3} ; t ; p, q\right) \\
=\frac{(q ; q)^{n} \Gamma_{t}(t ; q)^{n}}{n !} \int_{C^{n}} \prod_{1 \leq i \leq n} \frac{\theta\left(z_{i} / w, t^{n-1} v_{0} v_{1} / w z_{i} ; q\right)}{\theta\left(t^{i-1} v_{0} / w, t^{i-1} v_{1} / w ; q\right)} \prod_{1 \leq i<j \leq n} \frac{\Gamma_{t}\left(t z_{i} z_{j} / q, z_{i} z_{j}, t\left(z_{i} / z_{j}\right)^{ \pm 1} ; q\right)}{\Gamma_{t}\left(z_{i} z_{j} / q, z_{i} z_{j} / t,\left(z_{i} / z_{j}\right)^{ \pm 1} ; q\right)} \\
\prod_{1 \leq i \leq n}\left(1-z_{i}^{2} / q\right) \Gamma_{t}\left(v_{0} z_{i} / q, v_{1} z_{i} / q, v_{0} / z_{i}, v_{1} / z_{i} ; q\right) \prod_{0 \leq r \leq 2 m+3} \frac{\Gamma_{t}\left(z_{i} / u_{r} ; q\right)}{\Gamma_{t}\left(z_{i} u_{r} ; q\right)} \frac{d z_{i}}{2 \pi \sqrt{-1} z_{i}}
\end{aligned}
$$

where the contour $C$ is chosen so that for all $i \geq 0$, it contains the points and contours

$$
q^{i} v_{0}, q^{i} v_{1}, \quad q^{i} t C,
$$


and excludes the points and contours

$$
q^{1-i} / v_{0}, q^{1-i} / v_{1}, u_{r} / q^{i}, \quad q^{1-i} / t C, q^{-i} C / t,
$$

assuming such a contour exists.

Remark. It is easy to verify that there exist choices of the parameters for which a circular contour of radius $q^{1 / 2}$ satisfies the given conditions, and thus the integral on the right has a well-defined meromorphic extension to general parameters (and the limit will continue to hold); the only question is whether this can be obtained from a domain of integration of the form $C^{n}$.

Corollary 5.11. When $m=0$, the above trigonometric integral evaluates to

$$
\prod_{0 \leq i<n} \frac{\Gamma_{t}\left(t^{i+1}, t^{i} v_{0} v_{1} / q ; q\right) \prod_{0 \leq r \leq 3} \Gamma_{t}\left(t^{i} v_{0} / u_{r}, t^{i} v_{1} / u_{r} ; q\right)}{\prod_{0 \leq r<s \leq 3} \Gamma_{t}\left(t^{-i} u_{r} u_{s} ; q\right)}
$$

We also obtain a transformation.

Corollary 5.12. When $m=1$, the trigonometric integral of Theorem 5.8 is equal to

$$
\prod_{0 \leq i<n} \frac{\prod_{0 \leq r<s \leq 5} \Gamma\left(t^{i} u_{r} u_{s} ; p, q\right)}{\prod_{0 \leq r \leq 5} \Gamma\left(v_{0} / t^{i} u_{r}, v_{1} / t^{i} u_{r} ; p, q\right) \Gamma\left(v_{0} v_{1} / t^{i} q ; p, q\right)}
$$

times the image of the trigonometric integral of Theorem 5.10 under the specialization $u_{i} \mapsto t^{(n-1) / 2} u_{i}, v_{i} \rightarrow$ $t^{-(n-1) / 2} v_{i}$.

There are other transformations relating these integrals, but all can be obtained by applying the above transformation to one or both sides of a transformation of the integral of Theorem 5.8 alone.

Similarly, taking $a=p^{1 / 2}$ above, we obtain the limit

Theorem 5.13. For any nonzero parameters $t_{0}, t_{1}, u_{0}, \ldots, u_{m+1}, v_{0}, \ldots, v_{m}, p, q$ with

$$
0<|p|,|q|<1, \quad t^{2 n-2} t_{0} t_{1}=\prod_{0 \leq r \leq m+2} u_{r} \prod_{0 \leq r \leq m} v_{r}
$$

and any complex parameter $w \neq 0$, we have the identity

$$
\begin{gathered}
\lim _{p \rightarrow 0} \prod_{0 \leq i<n} \theta\left(t^{i} t_{0} t_{1} / p^{1 / 2} ; q\right) I I_{B C_{n}}^{(m)}\left(t_{0} / p^{1 / 4}, t_{1} / p^{1 / 4}, p^{1 / 4} / u_{0}, \ldots, p^{1 / 4} / u_{m+2}, p^{3 / 4} q / v_{0}, \ldots, p^{3 / 4} q / v_{m} ; t ; p, q\right) \\
=\frac{(q ; q)^{n} \Gamma_{t}(t ; q)^{n}}{n !} \int_{C^{n}} \prod_{1 \leq i \leq n} \frac{\theta\left(z_{i} / w, t^{n-1} t_{0} t_{1} / w z_{i} ; q\right)}{\theta\left(t^{i-1} t_{0} / w, t^{i-1} t_{1} / w ; q\right)} \prod_{1 \leq i<j \leq n} \frac{\Gamma_{t}\left(t\left(z_{i} / z_{j}\right)^{ \pm 1} ; q\right)}{\Gamma_{t}\left(\left(z_{i} / z_{j}\right)^{ \pm 1} ; q\right)} \\
\prod_{1 \leq i \leq n} \frac{\Gamma_{t}\left(t_{0} / z_{i}, t_{1} / z_{i} ; q\right) \prod_{0 \leq r \leq m+2} \Gamma_{t}\left(z_{i} / u_{r} ; p, q\right)}{\prod_{0 \leq r \leq m} \Gamma_{t}\left(z_{i} v_{r} ; p, q\right)} \frac{d z_{i}}{2 \pi \sqrt{-1} z_{i}},
\end{gathered}
$$

where the contour $C$ is chosen so that for all $j \geq 0$, it contains the points and contours

$$
q^{j} t_{0}, q^{j} t_{1}, \quad q^{j} t C
$$

and excludes the points and contours

$$
u_{r} / q^{j}, \quad C / q^{j} t
$$


Of course, with the Type II integral, we are particularly interested in the effect of multiplying the integrand for $m=0$ by the biorthogonal functions. Note that since the integral is taken over a compact curve in each case, the limiting relation will continue to hold as long as the limit of biorthogonal functions exists, and (more difficult) the revised contour conditions are satisfiable in the limit. The primary constraint is that we may only consider $p$-abelian biorthogonal functions, since otherwise the contour must contain at least one point converging to $\infty$ as $p \rightarrow 0$. For the first two limits, there is no difficulty with convergence of the biorthogonal function. Indeed, the $p$-abelian biorthogonal functions satisfy the further identities

$$
\begin{aligned}
\tilde{\mathcal{R}}_{0 \lambda}^{(n)} & \left(\ldots p^{ \pm 1 / 2} z_{i} \ldots ; p^{1 / 2} t_{0}: p^{1 / 2} t_{1}, p^{-1 / 2} t_{2}, p^{-1 / 2} t_{3} ; p^{1 / 2} u_{0}, p^{-1 / 2} u_{1} ; t ; p, q\right) \\
& =\tilde{\mathcal{R}}_{0 \lambda}^{(n)}\left(\ldots p^{ \pm 1 / 2} z_{i} \ldots ; p^{-1 / 2} t_{0}: p^{-1 / 2} t_{1}, p^{1 / 2} t_{2}, p^{1 / 2} t_{3} ; p^{1 / 2} u_{0}, p^{-1 / 2} u_{1} ; t ; p, q\right) \\
& =\tilde{\mathcal{R}}_{0 \lambda}^{(n)}\left(\ldots z_{i} \ldots ; t_{0}: t_{1}, t_{2}, t_{3} ; u_{0}, u_{1} ; t ; p, q\right)
\end{aligned}
$$

and thus in each case the relevant limit of biorthogonal functions is the same.

For the $a=\sqrt{p}$ limit, the situation is more delicate, but we find that if $t^{2 n-2} a_{0} a_{1} b_{0} b_{1} c d=q$, then we have a well-defined limit

$$
\begin{aligned}
R_{\lambda ; A S-I}^{(n)}( & \left(\ldots, z_{i}, \ldots ; a_{0}: a_{1}, b_{0}, b_{1} ; c, d ; q, t ; p\right) \\
& :=\lim _{p \rightarrow 0} \tilde{\mathcal{R}}_{0 \lambda}^{(n)}\left(\ldots p^{-1 / 4} z_{i} \ldots ; p^{-1 / 4} a_{0}: p^{-1 / 4} a_{1}, p^{1 / 4} b_{0}, p^{1 / 4} b_{1} ; p^{1 / 4} c, p^{3 / 4} d ; t ; p, q\right) \\
& =\lim _{p \rightarrow 0} \tilde{\mathcal{R}}_{0 \lambda}^{(n)}\left(\ldots p^{-1 / 4} / z_{i} \ldots ; p^{1 / 4} a_{0}: p^{1 / 4} a_{1}, p^{-1 / 4} b_{0}, p^{-1 / 4} b_{1} ; p^{3 / 4} c, p^{1 / 4} d ; t ; p, q\right) .
\end{aligned}
$$

This is a multivariate analogue of the biorthogonal rational functions of Al-Salam and Ismail [1]. More precisely, by specializing the $a=\sqrt{p}$ limit appropriately (in particular, $w=c^{-1}$ ), we find that the functions

$$
R_{\lambda ; A S-I}^{(n)}\left(\ldots, z_{i}, \ldots ; a_{0}: a_{1}, b_{0}, b_{1} ; c, d ; q, t ; p\right) \text { and } R_{\mu ; A S-I}^{(n)}\left(\ldots, 1 / z_{i}, \ldots ; b_{0}: b_{1}, a_{0}, a_{1} ; d, c ; q, t ; p\right)
$$

are biorthogonal with respect to the density

$$
\prod_{1 \leq i<j \leq n} \frac{\Gamma_{t}\left(t\left(z_{i} / z_{j}\right)^{ \pm 1} ; q\right)}{\Gamma_{t}\left(\left(z_{i} / z_{j}\right)^{ \pm 1} ; q\right)} \prod_{1 \leq i \leq n} \frac{\Gamma_{t}\left(a_{0} / z_{i}, a_{1} / z_{i}, b_{0} z_{i}, b_{1} z_{i} ; q\right)}{\Gamma_{t}\left(q / c z_{i}, q z_{i} / d, t^{n-1} a_{0} a_{1} c / z_{i}, t^{n-1} b_{0} b_{1} d z_{i} ; q\right)}
$$

which becomes Al-Salam and Ismail's density when $t^{n-1} a_{0} a_{1} c=q^{1 / 2}=t^{n-1} b_{0} b_{1} d$ and $n=1$. The constraints on the contour are independent of $\lambda, \mu, c$ and $d$, and are simply that $C$ must contain the points $q^{i} a_{r}$ and the contours $q^{i} t C$, and exclude the points $1 / q^{i} b_{r}$ and the contours $C / q^{i} t$. If we then take $a_{1}, b_{1} \rightarrow 0$ and set $a_{0}=b_{0}=q^{1 / 2}$, we obtain polynomials biorthogonal with respect to the density

$$
\prod_{1 \leq i<j \leq n} \frac{\Gamma_{t}\left(t\left(z_{i} / z_{j}\right)^{ \pm 1} ; q\right)}{\Gamma_{t}\left(\left(z_{i} / z_{j}\right)^{ \pm 1} ; q\right)}
$$

these are, of course, simply the ordinary Macdonald polynomials [11, up to a suitable normalization. That these arise as limits of the biorthogonal functions is not particularly new (since they are limits of Koornwinder polynomials); what is new is that a limit exists that respects the inner product.

It should be possible to obtain similar limits in the $A_{n}$ case; since the contour conditions are significantly more complicated in that case, however, we mention only the identity which presumably plays the role of Lemma 
5.2 in this case:

$$
\begin{aligned}
\operatorname{symm}_{S_{n+1}} \frac{\prod_{0 \leq i \leq n} \theta\left(x z_{i} / \prod_{0 \leq j<i} v_{j} \prod_{i<j \leq n} u_{j} ; q\right) \prod_{0 \leq j<i} \theta\left(v_{j} z_{i} ; q\right) \prod_{i<j \leq n} \theta\left(u_{j} z_{i} ; q\right)}{\prod_{0 \leq i<j \leq n} z_{j} \theta\left(z_{i} / z_{j} ; q\right) u_{j} \theta\left(v_{i} / u_{j} ; q\right)} & =\theta\left(x \prod_{0 \leq j \leq n} z_{j} ; q\right) \prod_{1 \leq i \leq n} \theta\left(x / \prod_{0 \leq j<i} v_{j} \prod_{i \leq j \leq n} u_{j} ; q\right),
\end{aligned}
$$

a special case of Theorem 4.4 of [17].

\section{Rational limits}

The rational limit is most naturally viewed as a combination of the hyperbolic and trigonometric limits, and thus in particular requires both the asymptotic calculations from the hyperbolic case and the symmetry breaking from the trigonometric case. In addition, it can be reached by taking $\omega_{2} \rightarrow 0$ in the hyperbolic case, or $q \rightarrow 1$ in the trigonometric or elliptic cases. We consider the limit from the elliptic level, as the other levels introduce no further complications. In each case, the integrand factors as a product of $q$-theta functions and functions to which Corollary 2.15 applies; the exponential behaviour of the integrand comes only from the former.

Theorem 6.1. For $\Im(\omega)>0, \sum_{0 \leq r \leq 2 n+m+2} \mu_{r}=\sum_{0 \leq r \leq m} \nu_{r}$,

$$
\begin{aligned}
\lim _{v \rightarrow 0^{+}} & e(-n(2 n+3) / 24 v \omega)((p ; p) \sqrt{2 \pi v \omega / \sqrt{-1}})^{n(2 n+3)} \\
& I_{B C_{n}}^{(m)}\left(e\left(v \mu_{0}\right), \ldots, e\left(v \mu_{2 n+m+2}\right), p e\left(v\left(\omega-\nu_{0}\right)\right), \ldots, p e\left(v\left(\omega-\nu_{m}\right)\right) ; p, e(v \omega)\right) \\
& =\frac{(\sqrt{2 \pi} \omega / \sqrt{-1})^{-n}}{2^{n} n !} \int_{C^{n}} \prod_{1 \leq i<j \leq n} \Gamma_{r}\left( \pm x_{i} \pm x_{j} ; \omega\right)^{-1} \prod_{1 \leq i \leq n} \frac{\prod_{0 \leq r \leq 2 n+m+2} \Gamma_{r}\left(\mu_{r} \pm x_{i} ; \omega\right)}{\Gamma_{r}\left( \pm 2 x_{i} ; \omega\right) \prod_{0 \leq r \leq m} \Gamma_{r}\left(\nu_{r} \pm x_{i} ; \omega\right)} d x_{i},
\end{aligned}
$$

where $C$ is a contour agreeing with $\mathbb{R}$ outside a compact set, and separating the points of the form $\mu_{r}+j \omega$, $j \geq 0$ from the points of the form $-\mu_{r}-j \omega, j \geq 0$.

Proof. We consider the case in which the original contour is the unit circle; deformed cases are analogous. As in the hyperbolic case, we make the change of variables $z_{i}=e\left(x_{i}\right)$ and integrate over $[-1 / 2,1 / 2]^{n}$. If we divide the integrand by

$$
\prod_{1 \leq i \leq j \leq n} \theta\left(e\left(x_{i}+x_{j}\right) ; e(v \omega)\right) \prod_{1 \leq i<j \leq n} \theta\left(e\left(x_{i}-x_{j}\right) ; e(v \omega)\right) \prod_{1 \leq i \leq n} \theta\left(e\left(v \omega / 2+x_{i}\right) ; e(v \omega)\right)^{-2 n-2}
$$

the remaining factors of the integrand are controlled by Corollary 2.15 to have at worst polynomial growth in $v$, and thus the exponential behavior of the theta functions dominates. In particular, up to polynomial factors, the integrand satisfies the bound

$$
O\left(e\left((-1 / 2 v \omega)\left[\sum_{1 \leq i \leq n} 2(n+1) \vartheta\left(x_{i}\right)-\sum_{1 \leq i<j \leq n} \vartheta\left(x_{i} \pm x_{j}\right)-\sum_{1 \leq i \leq n} \vartheta\left(2 x_{i}\right)\right]\right)\right)
$$

which decays exponentially unless $x_{1}, \ldots, x_{n}=o(1)$. We can thus restrict the integral to $[-1 / 4,1 / 4]^{n}$ and rescale the variables by $v$. The result then follows from Corollary 2.14, 
The other case is more complicated, in that the exponential contribution to the asymptotics is not enough to properly localize the integral.

Theorem 6.2. For $\Im(\omega)>0, \sum_{0 \leq r \leq 2 n+m+2} \mu_{r}=\sum_{0 \leq r \leq m} \nu_{r}$,

$$
\begin{gathered}
\lim _{v \rightarrow 0^{+}} e\left(-\left(m^{2}+2 m\right) / 24 v \omega\right)((p ; p) \sqrt{2 \pi v \omega / \sqrt{-1}})^{2 m^{2}+3 m} \prod_{0 \leq r<s \leq m} \theta\left(e\left(v\left(\nu_{r}+\nu_{s}-\omega\right)\right) / p ; e(v \omega)\right) \\
I_{B C_{m}}^{(n)}\left(\sqrt{p} e\left(v\left(\omega / 2-\mu_{0}\right)\right), \ldots, \sqrt{p} e\left(v\left(\omega / 2-\mu_{2 n+m+2}\right)\right), \frac{e\left(v\left(\nu_{0}-\omega / 2\right)\right)}{\sqrt{p}}, \ldots, \frac{e\left(v\left(\nu_{m}-\omega / 2\right)\right)}{\sqrt{p}} ; p, e(v \omega)\right) \\
=\frac{(\sqrt{2 \pi} \omega / \sqrt{-1})^{-m}}{m !} \int_{C^{m}} \frac{\theta_{h}\left(\sum_{0 \leq r \leq m} \nu_{r}-w-\sum_{1 \leq i \leq m} x_{i} ; \omega\right)}{\prod_{1 \leq i \leq m} \theta_{h}\left(x_{i}-w ; \omega\right)^{-1} \prod_{0 \leq r \leq m} \theta_{h}\left(\nu_{r}-w ; \omega\right)} \frac{\prod_{1 \leq i \leq j \leq m}\left(\left(x_{i}+x_{j}\right) / \omega-1\right)}{\prod_{1 \leq i<j \leq m} \Gamma_{r}\left( \pm\left(x_{i}-x_{j}\right) ; \omega\right)} \\
\prod_{1 \leq i \leq m} \prod_{0 \leq r \leq 2 n+m+2} \frac{\Gamma_{r}\left(x_{i}-\mu_{r} ; \omega\right)}{\Gamma_{r}\left(x_{i}+\mu_{r} ; \omega\right)} \prod_{0 \leq r \leq m} \Gamma_{r}\left(\nu_{r}+x_{i}-\omega, \nu_{r}-x_{i} ; \omega\right) d x_{i},
\end{gathered}
$$

where $C$ is a contour agreeing with $\mathbb{R}$ outside a compact set, and separating the points of the form $\nu_{r}+j \omega$, $j \geq 0$ from the points of the form $\mu_{r}-j \omega,-\nu_{r}-(j-1) \omega, j \geq 0$.

Proof. We begin with the integral of Lemma 5.3, replacing the extra parameter by $e(v w)$. The exponential factor in the asymptotics of the resulting elliptic integrand is

$$
e\left(\left(\vartheta\left(\sum_{1 \leq i \leq m} x_{i}\right)+\sum_{1 \leq i<j \leq m} \vartheta\left(x_{i}-x_{j}\right)-m \sum_{1 \leq i \leq m} \vartheta\left(x_{i}\right)\right) / 2 v \omega\right),
$$

and thus the integrand is exponentially small unless the sequence $x_{1}, \ldots, x_{m}$ in $\mathbb{R} / \mathbb{Z}$ interlaces (or nearly interlaces) with the all-zero sequence. More precisely, if we split the integral into $2^{m}$ integrals based on the decomposition $\mathbb{R} / \mathbb{Z}=[-1 / 4,1 / 4] \cup[1 / 4,3 / 4]$, then any piece with more than one $[1 / 4,3 / 4]$ factor contributes an exponentially small amount. For the pieces with exactly one $[1 / 4,3 / 4]$ factor, we find that upon rescaling the $[-1 / 4,1 / 4]$ variables, the resulting integrand has order $O\left(v^{2}\right)$ and is integrable; thus those pieces again contribute a negligible amount to the limit. We may thus restrict our attention to $[-1 / 4,1 / 4]^{n}$, or equivalently (up to $O(v)$ ), the integral over $[-1 / 4 v, 1 / 4 v]$ of the rational limit integrand. The omitted tails are again either exponentially small or have integral of order $O\left(v^{2}\right)$, so the result follows.

Corollary 6.3. The rational integral of Theorem 6.1 is equal to

$$
\prod_{0 \leq r<s \leq 2 n+m+2} \Gamma_{r}\left(\mu_{r}+\mu_{s} ; \omega\right) \prod_{\substack{0 \leq r \leq 2 n+m+2 \\ 0 \leq s \leq m}} \Gamma_{r}\left(\nu_{s}-\mu_{r} ; \omega\right)^{-1} \prod_{0 \leq r<s \leq m} \Gamma_{r}\left(\nu_{r}+\nu_{s}-\omega ; \omega\right)^{-1}
$$

times the rational integral of Theorem 6.2.

For the Type II integral, again the first case is straightforward.

Theorem 6.4. For any parameters $\mu_{0}, \ldots, \mu_{m+4}, \nu_{0}, \ldots, \nu_{m}, \omega, \tau$ satisfying

$$
\Im(\omega), \Im(\tau)>0, \quad(2 n-2) \tau+\prod_{0 \leq r \leq m+4} \mu_{r}=\prod_{0 \leq r \leq m} \nu_{r}
$$


we have the limit

$$
\begin{aligned}
\lim _{v \rightarrow 0^{+}} & e(-n / 4 v \omega)((p ; p) \sqrt{2 \pi v \omega / \sqrt{-1}})^{2 n(2 n-3) \tau / \omega+6 n} \\
& I I_{B C_{n}}^{(m)}\left(e\left(v \mu_{0}\right), \ldots, e\left(v \mu_{m+4}\right), p e\left(v\left(\omega-\nu_{0}\right)\right), \ldots, p e\left(v\left(\omega-\nu_{m}\right)\right) ; e(v \tau) ; p, e(v \omega)\right) \\
& =\frac{\Gamma_{r}(\tau ; \omega)^{n}}{(\sqrt{2 \pi} \omega / \sqrt{-1})^{n} 2^{n} n !} \int_{C^{n}} \prod_{1 \leq i<j \leq n} \frac{\Gamma_{r}\left(\tau \pm x_{i} \pm x_{j} ; \omega\right)}{\Gamma_{r}\left( \pm x_{i} \pm x_{j} ; \omega\right)} \prod_{1 \leq i \leq n} \frac{\prod_{0 \leq r \leq m+4} \Gamma_{r}\left(\mu_{r} \pm x_{i} ; \omega\right)}{\Gamma_{r}\left( \pm 2 x_{i} ; \omega\right) \prod_{0 \leq r \leq m} \Gamma_{r}\left(\nu_{r} \pm x_{i} ; \omega\right)} d x_{i}
\end{aligned}
$$

where the contour $C$ agrees with $\mathbb{R}$ outside a compact set, satisfies $C=-C$ and for all $i \geq 0$, contains the points $i \omega+\mu_{r}$ as well as the contour $i \omega+\tau+C$.

Proof. The exponential factor in the asymptotics of the integrand is

$$
O\left(e\left(\sum_{i}\left(\vartheta\left(2 x_{i}\right)-4 \vartheta\left(x_{i}\right)\right) / 2 v \omega\right)\right)
$$

which is exponentially small unless $x_{i} \equiv 0$. The limit follows as above.

The nonsymmetric Type II integral has even worse behavior than the nonsymmetric Type I case, however.

Theorem 6.5. Let $\mu_{0}, \ldots, \mu_{2 m+3}, \nu_{0}, \nu_{1}, \omega, \tau, p$ be parameters such that $|p|<1, \Im(\omega), \Im(\tau)>0$, and

$$
(2 n-2) \tau+\nu_{0}+\nu_{1}=\sum_{r} \mu_{r}
$$

as well as the convergence condition $\Re(\tau / \omega)>-1 / n$. Then we have the limit

$$
\begin{gathered}
\lim _{v \rightarrow 0} e(-n / 4 v \omega)((p ; p) \sqrt{2 \pi v \omega / \sqrt{-1}})^{2 n(2 n-3) \tau / \omega+6 n} \prod_{0 \leq i<n} \theta\left(e\left(v\left(i \tau+\nu_{0}+\nu_{1}-\omega\right) / p\right) ; q\right) \\
\quad I_{B C_{n}}^{(m)}\left(\frac{e\left(v\left(\nu_{0}-\omega / 2\right)\right.}{\sqrt{p}}, \frac{e\left(v\left(\nu_{1}-\omega / 2\right)\right.}{\sqrt{p}}, \sqrt{p} e\left(v\left(\omega / 2-\mu_{0}\right)\right), \ldots, \sqrt{p} e\left(v\left(\omega / 2-\mu_{2 m+3}\right)\right) ; e(v \tau) ; p, e(v \omega)\right) \\
=\frac{\Gamma_{r}(\tau ; \omega)^{n}}{(\sqrt{2 \pi} \omega / \sqrt{-1})^{n} n !} \int_{C^{n}} \prod_{1 \leq i<j \leq n} \frac{\Gamma_{r}\left(\tau+x_{i}+x_{j}-\omega, x_{i}+x_{j}, \tau+x_{i}-x_{j}, \tau+x_{j}-x_{i} ; \omega\right)}{\Gamma_{r}\left(x_{i}+x_{j}-\omega, x_{i}+x_{j}-\tau, x_{i}-x_{j}, x_{j}-x_{i} ; \omega\right)} \\
\prod_{1 \leq i \leq n}\left(2 x_{i} / \omega-1\right) \Gamma_{r}\left(\nu_{0}+x_{i}-\omega, \nu_{1}+x_{i}-\omega, \nu_{0}-x_{i}, \nu_{1}-x_{i} ; \omega\right) \\
\frac{\theta_{h}\left(x_{i}-w,(n-1) \tau+\nu_{0}+\nu_{1}-w-x_{i} ; \omega\right)}{\theta_{h}\left((i-1) \tau+\nu_{0}-w,(i-1) \tau+\nu_{1}-w ; \omega\right)} \prod_{0 \leq r \leq 2 m+3} \frac{\Gamma_{r}\left(x_{i}-\mu_{r} ; \omega\right)}{\Gamma_{r}\left(x_{i}+\mu_{r} ; \omega\right)} d x_{i},
\end{gathered}
$$

where the contour $C$ is chosen so that for all $i \geq 0$, it contains the points and contours

$$
i \omega+\nu_{0}, i \omega+\nu_{1}, \quad i \omega+\tau+C,
$$

and excludes the points and contours

$$
(1-i) \omega-\nu_{0},(1-i) \omega-\nu_{1}, \mu_{r}-i \omega, \quad(1-i) \omega-\tau-C, C-i \omega-\tau,
$$

assuming such a contour exists. 
Proof. The exponential factors in the asymptotics of the elliptic integrand actually cancel completely, with the result that the integrand has polynomial asymptotics. The result will follow from dominated convergence if we can show that the rational integrand converges.

For the rational tails, we find that (assuming $C=\omega / 2+\mathbb{R}$ for simplicity) the integrand converges iff the integral

$$
\int_{\mathbb{R}^{n}} \prod_{1 \leq i<j \leq n}\left|x_{i}^{2}-x_{j}^{2}\right|^{2 \Re(\tau / \omega)} \prod_{1 \leq i \leq n}\left|x_{i}+\sqrt{-1} \epsilon\right|^{-4(n-1) \Re(\tau / \omega)-3} d x_{i}
$$

converges for $\epsilon>0$; equivalently, via the change of variables $y_{i}=x_{i}^{2} / \epsilon^{2}$, the theorem reduces to the convergence of

$$
\int_{[0, \infty)^{n}} \prod_{1 \leq i<j \leq n}\left|y_{i}-y_{j}\right|^{2 \Re(\tau / \omega)} \prod_{1 \leq i \leq n}\left|1+y_{i}\right|^{-2(n-1) \Re(\tau / \omega)-2} d y_{i} .
$$

Up to linear fractional transformation, this is an instance of the Selberg integral ([19], stated as Corollary 7.5 below), and thus converges as long as $\Re(\tau / \omega)>-1 / n$, as required.

\section{Classical limits}

The final limit we consider is that corresponding to the usual beta integral. Although the beta integral itself is generally viewed as the bottom level, this is in fact a somewhat misleading view, as the integrals we obtain are in fact still elliptic (involving powers of theta functions). For the $B C_{n}$ cases, a suitable change of variables exists that essentially eliminates the dependence on $p$, but the corresponding change of variables for the $A_{n}$ integral is much less obvious (if it exists at all). Furthermore, even for the beta case, the classical transformation analogue can only easily be reached by degenerating either the hyperbolic or elliptic levels; the symmetry breaking of the trigonometric and rational cases introduces unnecessary complications.

Since the integrand involves powers of theta functions, there are in general some subtle issues involving choices of branch. It will thus be convenient to restrict to the case $p$ real, where the phases are easier to control. We have the following.

Lemma 7.1. Choose $p$ and $z$ such that $-1<p<1$ and $|z|=1$. Then the standard branch of $\log \theta(z ; p)$ satisfies

$$
\begin{aligned}
\log \theta(z ; p) & =\log (-z) / 2+\log |\theta(z ; p)|, \\
\log \theta\left(p^{1 / 2} z ; p\right) & =\log \left|\theta\left(p^{1 / 2} z ; p\right)\right| .
\end{aligned}
$$

Proof. We have

$$
\log \theta(z ; p)=\log (1-z)+\sum_{1 \leq i} \log \left(1-p^{i} z\right)+\log \left(1-p^{i} / z\right)
$$

taking the principal branch of the logarithm on the right-hand side. Now,

$$
\log \left(1-p^{i} z\right)+\log \left(1-p^{i} / z\right)=2 \log \left|1-p^{i} z\right|
$$

so it suffices to show that

$$
\log (1-z)=\log (-z) / 2+\log |1-z|
$$


which follows from the observation $(1-z) / \sqrt{-z}=|1-z|$. Similarly,

$$
\log \theta\left(p^{1 / 2} z ; p\right)=\sum_{0 \leq i} 2 \log \left|1-p^{i+1 / 2} z\right| .
$$

We will thus assume $-1<p<1$ in the sequel; note, however, that the case of more general $p$ can be obtained by replacing

$$
\begin{aligned}
|\theta(z ; p)|^{\kappa} & \mapsto(-z)^{\kappa / 2} \theta(z ; p)^{\kappa} \\
\left|\theta\left(p^{1 / 2} z ; p\right)\right|^{\kappa} & \mapsto \theta\left(p^{1 / 2} z ; p\right)^{\kappa} .
\end{aligned}
$$

In any event, we need all parameters to have absolute value $1,|p|^{1 / 2}$, or $|p|$ within $1+o(1)$ for this to work.

For the Type I $B C_{n}$ integral, it is particularly natural to take $2 m+2$ parameters to have norm $|p|^{1 / 2}$, at which point both sides of the transformation take the same form. More general cases could be considered, but appear to give rise to the same limiting identities, so we will restrict our attention to the simplest case.

Given points $x, y, z$ on the unit circle with $x, z$ distinct, $y \in[x, z]$ or equivalently $x \leq y \leq z$ indicates that $y$ is on the closed counterclockwise arc from $x$ to $z$, and similarly for open arcs.

Theorem 7.2. Let $a_{0}, \ldots, a_{n}, b_{0}, \ldots, b_{m}$ be points on the unit circle with

$$
1 \leq a_{0}<a_{1}<\cdots<a_{n} \leq-1,
$$

let $\omega$ be a point in the upper half-plane, and let $\alpha_{0}^{ \pm}, \ldots, \alpha_{n}^{ \pm}, \beta_{0}^{ \pm}, \ldots, \beta_{m}^{ \pm}$be parameters such that $\Re\left(\alpha_{r}^{ \pm}\right)>0$ and

$$
\sum_{0 \leq r \leq n} \alpha_{r}^{+}+\alpha_{r}^{-}=\sum_{0 \leq r \leq m} \beta_{r}^{+}+\beta_{r}^{-} .
$$

Then, writing $q=e(v \omega), \alpha_{r}=\alpha_{r}^{+}+\alpha_{r}^{-}, \beta_{r}=\beta_{r}^{+}+\beta_{r}^{-}$, we have

$$
\begin{aligned}
& \lim _{v \rightarrow 0^{+}} \frac{\Gamma_{e}\left(q^{\sum_{r} \alpha_{r}} ; p, q\right)}{\prod_{0 \leq r \leq n} \Gamma_{e}\left(q^{\alpha_{r}} ; p, q\right) \prod_{0 \leq r<s \leq n} \Gamma_{e}\left(q^{\alpha_{r}^{+}+\alpha_{s}^{+}} a_{r} a_{s}, q^{\alpha_{r}^{+}+\alpha_{s}^{-}} a_{r} / a_{s}, q^{\alpha_{r}^{-}+\alpha_{s}^{+}} a_{s} / a_{r}, q^{\alpha_{r}^{-}+\alpha_{s}^{-}} / a_{r} / a_{s} ; p, q\right)} \\
& I_{B C_{n}}^{(m)}\left(\ldots, q^{\alpha_{r}^{+}} a_{r}, q^{\alpha_{r}^{-}} / a_{r}, \ldots, \ldots, p^{1 / 2} q^{1 / 2-\beta_{r}^{+}} / b_{r}, p^{1 / 2} q^{1 / 2-\beta_{r}^{-}} b_{r}, \ldots ; p, q\right) \\
&=\prod_{0 \leq r<s \leq n}\left|\theta\left(a_{r} a_{s}^{ \pm 1} ; p\right)\right|^{1-\alpha_{r}-\alpha_{s}} \frac{\Gamma\left(\sum_{0 \leq r \leq n} \alpha_{r}\right)}{\prod_{0 \leq r \leq n} \Gamma\left(\alpha_{r}\right)}\left(2 \pi(p ; p)^{2}\right)^{n} \\
& \int_{z_{i} \in\left[a_{i-1}, a_{i}\right]} \prod_{1 \leq i<j \leq n}\left|\theta\left(z_{i} z_{j}^{ \pm 1} ; p\right)\right| \prod_{1 \leq i \leq n} \frac{\prod_{0 \leq r \leq n}\left|\theta\left(a_{r} z_{i}^{ \pm 1} ; p\right)\right|^{\alpha_{r}-1}}{\prod_{0 \leq r \leq m}\left|\theta\left(p^{1 / 2} b_{r} z_{i}^{ \pm 1} ; p\right)\right|^{\beta_{r}}} \frac{\left|\theta\left(z_{i}^{2} ; p\right)\right| d z_{i}}{2 \pi \sqrt{-1} z_{i}} .
\end{aligned}
$$

Proof. Using Lemma 3.3] we find that the integral decays exponentially outside the stated product of arcs (or images under the hyperoctahedral group). The result then follows by dominated convergence.

If we define $\phi(z)=-\theta(z ; p)^{2} / \theta(-z ; p)^{2}$, then

$$
\begin{aligned}
\phi(z)-\phi(w) & =\frac{\theta(-1 ; p)^{2} z \theta\left(w z^{ \pm 1} ; p\right)}{\theta(-z ; p)^{2} \theta(-w ; p)^{2}} \\
\phi^{\prime}(z) & =\frac{\theta(-1 ; p)^{2}(p ; p)^{2} \theta\left(z^{2} ; p\right)}{\theta(-z ; p)^{4}},
\end{aligned}
$$


so for $z$ in the arc $[1,-1]$,

$$
d \phi(z)=2 \pi(p ; p)^{2} \frac{\theta(-1 ; p)^{2}}{|\theta(-z ; p)|^{4}} \frac{\left|\theta\left(z^{2} ; p\right)\right| d z}{2 \pi \sqrt{-1} z}
$$

and

$$
\begin{gathered}
\left|\theta\left(a z^{ \pm 1} ; p\right)\right|^{\kappa}=|\phi(z)-\phi(a)|^{\kappa} \frac{|\theta(-z ; p)|^{2 \kappa}|\theta(-a ; p)|^{2 \kappa}}{|\theta(-1 ; p)|^{2 \kappa}} \\
\left|\theta\left(p^{1 / 2} b z^{ \pm 1} ; p\right)\right|^{\kappa}=\left|\phi(z)-\phi\left(p^{1 / 2} b\right)\right|^{\kappa} \frac{|\theta(-z ; p)|^{2 \kappa}\left|\theta\left(-p^{1 / 2} b ; p\right)\right|^{2 \kappa}}{|\theta(-1 ; p)|^{2 \kappa}}
\end{gathered}
$$

Consequently, we can make a change of variables in the resulting transformation to obtain the following result of Dixon [6].

Corollary 7.3. For any parameters $a_{0}, \ldots, a_{n}, b_{0}, \ldots, b_{m}, \alpha_{0}, \ldots, \alpha_{n}, \beta_{0}, \ldots, \beta_{m}$ such that

$$
-b_{m}<-b_{m-1}<\cdots<-b_{0}<a_{0}<a_{1}<\cdots<a_{n}, \quad 0<\Re\left(\alpha_{r}\right), \Re\left(\beta_{r}\right), \quad \sum_{0 \leq r \leq n} \alpha_{r}=\sum_{0 \leq r \leq m} \beta_{r}
$$

we have the identity

$$
\begin{aligned}
& \frac{\prod_{0 \leq i<j \leq n}\left|a_{i}-a_{j}\right|^{1-\alpha_{i}-\alpha_{j}}}{\prod_{0 \leq i \leq n} \Gamma\left(\alpha_{i}\right) \prod_{0 \leq j \leq m}\left|a_{i}+b_{j}\right|^{\alpha_{i}}} \int_{x_{i} \in\left[a_{i-1}, a_{i}\right]} \prod_{1 \leq i<j \leq n}\left|x_{i}-x_{j}\right| \prod_{1 \leq i \leq n} \frac{\prod_{0 \leq j \leq n}\left|a_{j}-x_{i}\right|^{\alpha_{j}-1}}{\prod_{0 \leq j \leq m}\left|x_{i}+b_{j}\right|^{\beta_{j}}} d x_{i} \\
& =\frac{\prod_{0 \leq i<j \leq m}\left|b_{i}-b_{j}\right|^{1-\beta_{i}-\beta_{j}}}{\prod_{0 \leq i \leq m} \Gamma\left(\beta_{i}\right) \prod_{0 \leq j \leq n}\left|b_{i}+a_{j}\right|^{\beta_{i}}} \int_{x_{i} \in\left[b_{i-1}, b_{i}\right]} \prod_{1 \leq i<j \leq m}\left|x_{i}-x_{j}\right| \prod_{1 \leq i \leq m} \frac{\prod_{0 \leq j \leq m}\left|b_{j}-x_{i}\right|^{\beta_{j}-1}}{\prod_{0 \leq j \leq n}\left|x_{i}+a_{j}\right|^{\alpha_{j}}} d x_{i} .
\end{aligned}
$$

A similar argument gives the type II analogue.

Theorem 7.4. Let $a_{0}, a_{1}, b_{0}, \ldots, b_{m}$ be points on the unit circle with

$$
1 \leq a_{0}<a_{1} \leq-1
$$

let $\omega$ be a point in the upper half-plane, and let $\alpha_{0}^{ \pm}, \alpha_{1}^{ \pm}, \beta_{0}^{ \pm}, \ldots, \beta_{m}^{ \pm}, \tau$ be parameters such that

$$
\Re\left(\alpha_{0}^{ \pm}\right), \Re\left(\alpha_{1}^{ \pm}\right), \Re(\tau)>0, \quad 2(n-1) \tau+\alpha_{0}+\alpha_{1}=\sum_{0 \leq r \leq m} \beta_{r},
$$

where $\alpha_{r}=\alpha_{r}^{+}+\alpha_{r}^{-}, \beta_{r}=\beta_{r}^{+}+\beta_{r}^{-}$. Writing $q=e(v \omega)$, we have

$$
\begin{aligned}
\lim _{v \rightarrow 0^{+}} & \prod_{0 \leq i<n} \frac{\Gamma_{e}\left(q^{2(n-1) \tau+\alpha_{0}+\alpha_{1}-i \tau} ; p, q\right)}{\Gamma_{e}\left(q^{(i+1) \tau}, q^{i \tau+\alpha_{0}}, q^{i \tau+\alpha_{1}}, q^{i \tau+\alpha_{0}^{+}+\alpha_{1}^{+}} a_{0} a_{1}, q^{i \tau+\alpha_{0}^{+}+\alpha_{1}^{-}} a_{0} / a_{1}, q^{i \tau+\alpha_{0}^{-}+\alpha_{1}^{+}} a_{1} / a_{0}, q^{i \tau+\alpha_{0}^{-}+\alpha_{1}^{-}} / a_{0} a_{1} ; p, q\right)} \\
& I_{B C_{n}}^{(m)}\left(q^{\alpha_{0}^{+}} a_{0}, q^{\alpha_{0}^{-}} / a_{0}, q^{\alpha_{1}^{+}} a_{1}, q^{\alpha_{1}^{-}} / a_{1}, \ldots, p^{1 / 2} q^{1 / 2-\beta_{r}^{+}} / b_{r}, p^{1 / 2} q^{1 / 2-\beta_{r}^{-}} b_{r}, \ldots ; q^{\tau}, p, q\right) \\
= & \left|\theta\left(a_{0} a_{1}^{ \pm 1} ; p\right)\right|^{n-n(n-1) \tau-n \alpha_{0}-n \alpha_{1}} \prod_{0 \leq i<n} \frac{\Gamma\left(2(n-1) \tau+\alpha_{0}+\alpha_{1}-i \tau\right) \Gamma(\tau)}{\Gamma((i+1) \tau) \Gamma\left(i \tau+\alpha_{0}\right) \Gamma\left(i \tau+\alpha_{1}\right)} \\
& \frac{\left(2 \pi(p ; p)^{2}\right)^{n}}{n !} \int_{\left[a_{0}, a_{1}\right]^{n}} \prod_{1 \leq i<j \leq n}\left|\theta\left(z_{i} z_{j}^{ \pm 1} ; p\right)\right|^{2 \tau} \prod_{1 \leq i \leq n} \frac{\left|\theta\left(a_{0} z_{i}^{ \pm 1} ; p\right)\right|^{\alpha_{0}-1}\left|\theta\left(a_{1} z_{i}^{ \pm 1} ; p\right)\right|^{\alpha_{1}-1}}{\prod_{0 \leq j \leq m}\left|\theta\left(p^{1 / 2} b_{j} z_{i}^{ \pm 1} ; p\right)\right|^{\beta_{j}}} \frac{\left|\theta\left(z_{i}^{2} ; p\right)\right| d z_{i}}{2 \pi \sqrt{-1} z_{i}}
\end{aligned}
$$


Corollary 7.5. [19] For any real numbers $a_{0}, a_{1}, b$ with $-b<a_{0}<a_{1}$, and parameters $\alpha_{0}, \alpha_{1}, \tau$ with positive real part,

$$
\begin{aligned}
\frac{1}{n !} \int_{\left[a_{0}, a_{1}\right]^{n}} & \prod_{1 \leq i<j \leq n}\left|x_{i}-x_{j}\right|^{2 \tau} \prod_{\substack{1 \leq i \leq n\\
}} \frac{\left|a_{0}-x_{i}\right|^{\alpha_{0}-1}\left|a_{1}-x_{i}\right|^{\alpha_{1}-1}}{\left|b+x_{i}\right|^{2(n-1) \tau+\alpha_{0}+\alpha_{1}}} d x_{i} \\
= & \frac{\left|a_{0}-a_{1}\right|^{n(n-1) \tau+n \alpha_{0}+n \alpha_{1}-n}}{\left|a_{0}+b\right|^{n(n-1) \tau+n \alpha_{1}}\left|a_{1}+b\right|^{n(n-1) \tau+n \alpha_{0}}} \prod_{0 \leq i<n} \frac{\Gamma((i+1) \tau) \Gamma\left(i \tau+\alpha_{0}\right) \Gamma\left(i \tau+\alpha_{1}\right)}{\Gamma\left(2(n-1) \tau+\alpha_{0}+\alpha_{1}-i \tau\right) \Gamma(\tau)}
\end{aligned}
$$

Remark. In fact (as observed in [19]), the constraint that $\Re(\tau)>0$ is too strict, as can be seen from the fact that the right-hand side remains finite and positive as long as

$$
\Re(\tau)>-1 / n,-\Re\left(\alpha_{0}\right) /(n-1),-\Re\left(\alpha_{1}\right) /(n-1) .
$$

One can presumably weaken the conditions of Theorem 7.4 correspondingly.

Corollary 7.6. For any real numbers $a_{0}, a_{1}, b_{0}, b_{1}$ with $-b_{1}<-b_{0}<a_{0}<a_{1}$, and parameters $\alpha_{0}, \alpha_{1}, \beta_{0}$, $\beta_{1}, \tau$ with positive real part such that $\alpha_{0}+\alpha_{1}=\beta_{0}+\beta_{1}$, we have the transformation

$$
\begin{aligned}
\int_{\left[a_{0}, a_{1}\right]^{n}} \prod_{1 \leq i<j \leq n}\left|x_{i}-x_{j}\right|^{2 \tau} & \prod_{1 \leq i \leq n} \frac{\left|a_{0}-x_{i}\right|^{\alpha_{0}-1}\left|a_{1}-x_{i}\right|^{\alpha_{1}-1}}{\left|b_{0}+x_{i}\right|^{(n-1) \tau+\beta_{0}}\left|b_{1}+x_{i}\right|^{(n-1) \tau+\beta_{1}}} d x_{i} \\
& =C \int_{\left[b_{0}, b_{1}\right]^{n}} \prod_{1 \leq i<j \leq n}\left|x_{i}-x_{j}\right|^{2 \tau} \prod_{1 \leq i \leq n} \frac{\left|b_{0}-x_{i}\right|^{\beta_{0}-1}\left|b_{1}-x_{i}\right|^{\beta_{1}-1}}{\left|a_{0}+x_{i}\right|^{(n-1) \tau+\alpha_{0}}\left|a_{1}+x_{i}\right|^{(n-1) \tau+\alpha_{1}}} d x_{i}
\end{aligned}
$$

where

$$
C=\prod_{0 \leq i<n} \frac{\left|a_{0}+b_{0}\right|^{\alpha_{0}-\beta_{0}}\left|a_{0}+b_{1}\right|^{\alpha_{0}-\beta_{1}}\left|a_{1}+b_{0}\right|^{\alpha_{1}-\beta_{0}}\left|a_{1}+b_{1}\right|^{\alpha_{1}-\beta_{1}}}{\left|a_{0}-a_{1}\right|^{1-(n-1) \tau-\alpha_{0}-\alpha_{1}}\left|b_{0}-b_{1}\right|^{-1+(n-1) \tau+\beta_{0}+\beta_{1}}} \frac{\Gamma\left(i \tau+\alpha_{0}\right) \Gamma\left(i \tau+\alpha_{1}\right)}{\Gamma\left(i \tau+\beta_{0}\right) \Gamma\left(i \tau+\beta_{1}\right)} .
$$

The $A_{n}$ case is similar.

Theorem 7.7. Let $a_{0}, \ldots, a_{n}, b_{0}, \ldots, b_{m}, Z$ be points on the unit circle with

$$
a_{0}<\cdots<a_{n}<a_{n+1}:=a_{0},
$$

let $\omega$ be a point in the upper half-plane, and let $\alpha_{0}^{ \pm}, \ldots, \alpha_{n}^{ \pm}, \beta_{0}^{ \pm}, \ldots, \beta_{m}^{ \pm}$be parameters such that

$$
\Re\left(\alpha_{0}^{ \pm}\right), \ldots, \Re\left(\alpha_{n}^{ \pm}\right)>0, \quad \sum_{0 \leq r \leq n} \alpha_{r}-\sum_{0 \leq r \leq m} \beta_{r}
$$

where $\alpha_{r}=\alpha_{r}^{+}+\alpha_{r}^{-}, \beta_{r}=\beta_{r}^{+}+\beta_{r}^{-}$. Writing $q=e(v \omega)$, we have

$$
\begin{aligned}
& \lim _{v \rightarrow 0^{+}} \frac{\Gamma_{e}\left(q^{\sum_{r} \alpha_{r}} ; p, q\right)}{\prod_{0 \leq r, s \leq n} \Gamma_{e}\left(q^{\alpha_{r}^{-}+\alpha_{s}^{+}} a_{s} / a_{r} ; p, q\right) \Gamma_{e}\left(q^{\sum_{r} \alpha_{r}^{-}} Z / a_{0} \cdots a_{n} ; p, q\right) \Gamma_{e}\left(q^{\sum_{r} \alpha_{r}^{+}} a_{0} \cdots a_{n} / Z ; p, q\right)} \\
& I_{A_{n}}^{(m)}\left(Z \mid \ldots, q^{\alpha_{r}^{-}} / a_{r}, \ldots, \ldots, p^{1 / 2} q^{1 / 2-\beta_{r}^{-}} b_{r}, \ldots ; \ldots, q^{\alpha_{r}^{+}} a_{r}, \ldots, \ldots, p^{1 / 2} q^{1 / 2-\beta_{r}^{+}} / b_{r}, \ldots ; p, q\right) \\
&=\prod_{0 \leq r<s \leq n}\left|\theta\left(a_{s} / a_{r} ; p\right)\right|^{1-\alpha_{r}-\alpha_{s}}\left|\theta\left(Z / a_{0} \cdots a_{n} ; p\right)\right|^{1-\sum_{r} \alpha_{r}} \frac{\Gamma\left(\sum_{r} \alpha_{r}\right)}{\prod_{0 \leq r \leq n} \Gamma\left(\alpha_{r}\right)} \\
& \frac{\left(2 \pi(p ; p)^{2}\right)^{n}}{(n+1) !} \int_{\substack{\prod_{0 \leq i \leq n} z_{i}=Z \\
z_{i} \in\left[a_{i}, a_{i+1}\right]}} \prod_{0 \leq i<j \leq n}\left|\theta\left(z_{i} / z_{j} ; p\right)\right| \prod_{0 \leq i \leq n} \frac{\prod_{0 \leq r \leq n}\left|\theta\left(z_{i} / a_{r} ; p\right)\right|^{\alpha_{r}-1}}{\prod_{0 \leq r \leq m}\left|\theta\left(p^{1 / 2} b_{r} z_{i} ; p\right)\right|^{\beta_{r}}} \prod_{0 \leq i<n} \frac{d z_{i}}{2 \pi \sqrt{-1} z_{i}}
\end{aligned}
$$


Remark. The fact that the $n+m+2$ theta functions

$$
\prod_{0 \leq i \leq n} \theta\left(z_{i} / a_{r} ; p\right), \prod_{0 \leq i \leq n} \theta\left(p^{1 / 2} b_{r} z_{i} ; p\right)
$$

for fixed $\prod_{0 \leq i \leq n} z_{i}$ span an $n+1$-dimensional space is presumably relevant to finding an appropriate change of variables to eliminate the theta functions from the integrand. Clearly, though, the resulting computations would not give a trivial derivation of the above integral from a more traditional multivariate beta integral.

\section{References}

[1] W. A. Al-Salam and M. E. H. Ismail. A $q$-beta integral on the unit circle and some biorthogonal rational functions. Proc. Amer. Math. Soc., 121(2):553-561, 1994.

[2] G. E. Andrews, R. Askey, and R. Roy. Special functions, volume 71 of Encyclopedia of Mathematics and its Applications. Cambridge University Press, Cambridge, 1999.

[3] F. van de Bult, E. M. Rains, and J. Stokman. Properties of generalized univariate hypergeometric functions. Comm. Math. Phys., to appear.

[4] J. F. van Diejen and V. P. Spiridonov. Elliptic Selberg integrals. Internat. Math. Res. Notices, (20):1083$1110,2001$.

[5] J. F. van Diejen and V. P. Spiridonov. Unit circle elliptic beta integrals. Ramanujan J., 10(2):187-204, 2005.

[6] A. L. Dixon. On a generalisation of Legendre's formula $K E^{\prime}-(K-E) K^{\prime}=\frac{1}{2} \pi$. Proc. London Math. Soc. (2), 3:206-224, 1905.

[7] G. Frobenius. Über die elliptischen Functionen zweiter Art. J. für die reine und angew. Math., 93:53-68, 1882.

[8] G. Gasper and M. Rahman. Basic hypergeometric series, volume 96 of Encyclopedia of Mathematics and its Applications. Cambridge University Press, Cambridge, second edition, 2004.

[9] R. A. Gustafson. Some $q$-beta and Mellin-Barnes integrals with many parameters associated to the classical groups. SIAM J. Math. Anal., 23(2):525-551, 1992.

[10] T. H. Koornwinder. Jacobi functions as limit cases of $q$-ultraspherical polynomials. J. Math. Anal. Appl., 148(1):44-54, 1990.

[11] I. G. Macdonald. Symmetric Functions and Hall Polynomials. Oxford Univ. Press, Oxford, England, second edition, 1995.

[12] A. Narukawa. The modular properties and the integral representations of the multiple elliptic gamma functions. Adv. Math., 189(2):247-267, 2004. 
[13] B. Nassrallah and Mizan Rahman. Projection formulas, a reproducing kernel and a generating function for $q$-Wilson polynomials. SIAM J. Math. Anal., 16(1):186-197, 1985.

[14] F. W. J. Olver. Asymptotics and special functions. Academic Press, New York-London, 1974.

[15] E. M. Rains. Recurrences of elliptic hypergeometric integrals. In M. Noumi and K. Takasaki, editors, Elliptic integrable systems, volume 18 of Rokko Lectures in Mathematics, pages 183-199, 2005. arXiv:math.CA/0504285.

[16] E. M. Rains. Transformations of elliptic hypergeometric integrals. Ann. Math., to appear, 2008.

[17] H. Rosengren and M. Schlosser. Elliptic determinant evaluations and the Macdonald identities for affine root systems. Compos. Math., 142(4):937-961, 2006.

[18] S. N. M. Ruijsenaars. A generalized hypergeometric function satisfying four analytic difference equations of Askey-Wilson type. Comm. Math. Phys., 206(3):639-690, 1999.

[19] A. Selberg. Remarks on a multiple integral. Norsk Mat. Tidsskr., 26:71-78, 1944.

[20] V. P. Spiridonov. Classical elliptic hypergeometric functions and their applications. In M. Noumi and K. Takasaki, editors, Elliptic integrable systems, volume 18 of Rokko Lectures in Mathematics, pages 253287, 2005. arXiv:math.CA/0504285.

[21] J. V. Stokman. Generalized Cherednik-Macdonald identities. arXiv:0708.0934.

[22] J. V. Stokman. Hyperbolic beta integrals. Adv. Math., 190(1):119-160, 2005. 